\title{
Devonian (Emsian-Famennian) Palynomorphs
}

\author{
F. PARIS ${ }^{1}$, J. B. RICHARDSON ${ }^{2}$, W. RIEGEL ${ }^{3}$, M. STREEL ${ }^{4}$, \& M. VANGUESTAINE \\ ${ }^{1}$ Laboratoire de Paléontologie et de Stratigraphie, Université de Rennes 35042 Rennes Cedex, France. \\ ${ }^{2}$ Dept. of Palaeontology British Museum (Natural History) Cromwell Road, London SW7 5BD, UK. \\ ${ }^{3}$ Geologisches Paleontologisches Institut, Goldschmidtstrasse 3, 3400, Gottingen, W. Germany, \\ ${ }^{4}$ Laboratoire de Paléobotanique et Paléopalynologie, Université de Liège, 7 Place du Vingt Août, B 4000 Liège, Belgium.
}

\section{EMSIAN}

Miospore assemblages which can be positively assigned to the Early Devonian have, to date, only been recorded from core material from Well A1-33. Assemblages of Emsian age were recorded from the interval 8079 to $8091 \mathrm{ft}$. whilst assemblages from 8055 to $8076 \mathrm{ft}$. contain some taxa which are common in the Emsian elsewhere but are not diagnostic. Assemblages recovered from sediments in the interval 9700 to $9709 \mathrm{ft}$. in Well A1-33 were dominated by leiospheres together with smaller numbers of acanthomorph acritarchs. The age of this interval is unknown but it could be as old as Ordovician.

The Emsian assemblages recovered from the interval between 8079 to $8091 \mathrm{ft}$. contain a wide variety of azonate miospores together with species possessing an equatorial crassitude, specimens with zonate extensions are rare. Miospores with sculptural or structural modifications around their equators are relatively common i.e. ?Procoronaspora sp., Diatomozonotriletes sp. A. and Craspedispora craspeda. The presence in these assemblages of Emphanisporites annulatus, E. erraticus, E. rotatus, E. cf. decoratus, E. obscurus, Dibolisporites eifeliensis, D. cf. gibberosus, D. echinaceus, Apiculiretusispora brandtii together with small tripapillate miospores suggest an Early or Middle Emsian age to be probable. The assemblages are closely comparable with Emsian microfloras recorded in the Polignac Basin of Algeria (Jardiné \& Yapaudjian, 1968) and the Rhadames Basin of Western Libya (Massa \& Moreau-Benoit, 1976).

\section{EIF ELIAN}

Miospore assemblages of probable Eifelian age have been recovered from Well A1-37 from cored material at $10674 \mathrm{ft}$. The miospore populations which are extremely well preserved contain representatives of the following taxa:-Anapiculatisporites sp., Dibolisporites cf. bullatus, Hystricosporites spp., Emphanisporites spinaeformis, Diatomozonotriletes sp., Calyptosporites cf. velatus, $C$. cf. spinosus, Grandispora ef. libyensis, Samarisporites sp. A., S. cf. megaformis, ? Spinozonotriletes cf. naumovii, "Hymenozonotriletes" discors and Ancyrospora nettersheimensis. This assemblage is closely comparable with other previously reported Eifelian population from Algeria (Jardiné \& Yapaudjian, 1968), Saudi Arabia (Hemer \& Nygreen, 1966) and Western Libya (Massa \& Moreau-Benoit, 1976). Despite close similarity at generic level between the composition of the northeast Libyan assemblages with those from the Rhenish Middle Devonian of the Eifel region of Germany, comparison at specific level is almost impossible. The presence in the Libyan material of Ancyrospora nettersheimensis is however of interest since this species was found to be confined to the Late Emsian and Early Eifelian in the Eifel region (Riegel, 1974).

\section{GIVETIAN}

Givetian miospore assemblages have been encountered in several wells including A1-37, B1-31, E1-82 and G1-82. Diagnostic components in these assemblages include Acinosporites acanthomammillatus, $A$. macrospinosus, Dibolisporites echinaceus, Geminospora tuberculata, Grandispora inculta, G. libyensis, Rhabdosporites langii Verrucosisporites premnus and V. scurrus, and suggest an early Givetian age.

Chitinozoan populations have been recovered from the same horizons as the above miospore assemblages in Well A1-37 in the interval between 9251 to $9840 \mathrm{ft}$. The assemblage recovered from the sample at $9840 \mathrm{ft}$. is the most diversified in composition with representatives of Eisenackitina castor (sensu J. A. Legault, 1973) being the dominant component. Amongst the other species present are forms sometimes erroneously attributed to Angochitina devonica but here referred to as Gotlandochitina sp. B. In addition there are isolated individuals identified as Gotlandochitina milanensis, Ancyrochitina ?aequoris and Hoegisphaera glabra which were recorded as common components of the lower part of the AouinetOuenine II Formation in the Rhadames Basin of western Libya by Massa \& Moreau-Benoit (1976) which is also considered to be of Early Givetian age. Chitinozoan assemblages recovered from younger sediments at 9251 and $9290 \mathrm{ft}$. in Well A 1-37 are dominated by Fungochitina pilosa, a species which has previously been recorded from both Givetian and Frasnian sediments in the Rhadames Basin of western Libya, northern France and the U.S.A. (Taugourdeau, Ph. 1965; Collinson, C. \& Scott, A. J., 1958 and Urban, J. B. and Newport, R. L., 1973). 


\section{FRASNIAN}

Assemblages of Frasnian palynomorphs which have been recorded in Well C1-125 in the interval 10300 to $11000 \mathrm{ft}$. and in Well A1-115 between 8762 to $8784 \mathrm{ft}$. are characterised by the occurrence of the following miospore taxa:- Verrucosisporites bullatus, Samarisporites triangulatus, Ancyrospora langi and A. multifurcata. The presence in these assemblages of the acritarchs Horologinella quadrispina, $H$. horologia, Unellium winslowae and Villosacapsula globosa suggests by comparison with their previously known distribution in the Algerian Sahara (Jardiné et al., 1974) a Late Frasnian age.Villosacapsula globosa has previously been found in Belgium to be restricted to a narrow zone transitional to the Frasnian - Famennian boundary.

\section{FAMENNIAN}

Earliest Famennian palynomorphs have been recorded in Well A1-NC-92 in the interval 13400 to $13500 \mathrm{ft}$., where the diagnostic miospore Cyrtospora cristifer occurs in association with the distinctive FrasnianFamennian boundary association of acritarchs including Crassiangulina tesselita, Umbellasphaeridium deflandrei, Unellium winslowae, Veryhachium pannuceum and Villosacapsula globosa.

Late Famennian miospore populations are characterised by the presence of Leiotriletes libyensis, Rugospora flexuosa and abundant representatives of Spelaeotriletes cf. crustatus. Distinctive assemblages of latest Famennian (Strunian) age with the characteristic miospore Retispora lepidophyta accompanied by Knoxisporites literatus and Vallatisporites pusillites were recorded in Well C1-125 in the interval 9300 to $9700 \mathrm{ft}$. and in Well A1-37 between 8418 and $8421 \mathrm{ft}$. These miospore populations are accompanied by acritarchs of the sphaeromorph and acanthomorph type in which the genera Gorgonisphaeridium and Lophosphaeridium predominate.

\section{REFERENCES}

Arkhangelskaya, A. D. 1963. New Species of spores from Devonian deposits of the Russian Platform; Trudy VNIGNI, 37 (18-30), 122-153. (In Russian).

Arkhangelskaya, A. D. 1974. Spore-based zonation of the lower part of the Middle Devonian in central and eastern parts of the European U.S.S.R. (in Russian). Palynology of the Proterophyte and Palaeophyte, Pro. 3rd Int. Palynol. Conf. 56-59.

Collinson, C. \& Scott, A. J. 1958. Chitinozoan faunule of the Devonian Cedar Valley Formation. Illinois Geol. Survey., 247, 1-34.

Hemer, D. O. \& Nygreen, P. W. 1967. Devonian palynology of Saudi Arabia. Rev. Palaeobot. Palynol., 5, 51-61.

Jardiné, S. \& Yapaudjian, L. 1968. Lithostratigraphie et palynologie du Dévonien-Gothlandien gréseux du Bassin de Polignac (Sahara). Rev. Inst. Français Pétrole, 23, 439-469.

Jardiné, S., Combaz, A., Magloire, L., Peniguel, G. \& Vachey, G. 1972. Acritarches du Silurien terminal et du Dévonien du Sahara Algérien. C.R. 7th Congr. Int. Carb. Strat. Geol. Krefeld, 1971, 1, 295-311.

Jardiné, S., Combaz, A., Magloire, L., Peniguel, G. \& Vachey, G. 1974. Distribution stratigraphique des Acritarches dans le Paléozoique du Sahara Algérien. Rev. Palaeobot. Palynol. 18, 99-129.

Legault, J. A. 1973. Chitinozoa and Acritarcha of the Hamilton Formation (Middle Devonian), South-Western Ontario. Geol. Surv. Canada Bull., 221, 1-103.

\section{Explanation of Plate 17}

Figs. $1-8$ are $\times 500$; figs. $9-12$ are $\times 1000$

Fig. 1. Dibolisporites sp. A. A1-33, 8064-8067ft., Slide 711 A, Q32, AGC 181.

Fig. 2. Retusotriletes sp. A. A1-33, 8088-8091 ft., Slide 717A, M37/2 - M38/1, AGC 182.

Fig. 3. Apiculiretusispora cf. brandtii Streel, 1964. A1-33, 8088-8091 ft., Slide 718A, J33, AGC 183.

Fig. 4. ?Granulatisporites sp. A (?contaminant). A1-33, 8064-8067ft., Slide 711A, H33/3, AGC 184.

Fig. 5. Procoronaspora sp. A1-33, 8079-8082 ft., Slide 715B, 041/3, AGC 185.

Fig. 6. Diatomozonotriletes sp. A. A1-33, 8079-8082 ft., Slide 715B, F44/2, AGC 186.

Fig. 7. Emphanisporites cf. obscurus McGregor, 1961. A1-33, 8082-8085ft., Slide 716A, R30/4, AGC 187.

Fig. 8. Emphanisporites erraticus (Eisenack, 1944). A1-33, 8088-8091 ft., Slide 718A, 040/4 - 041/3, AGC 188.

Fig. 9. ?Camarozonotriletes sp. A1-33, 8085-8088ft., Slide 717A, 041/4, AGC 189.

Fig. 10. Aneurospora sp. A1-33, 8061-8064 ft., Slide 710A, K47/2, AGC 190.

Fig. 11. Emphanisporites cf. decoratus Allen, 1965. A1-33, 8085-8088ft., Slide 717A, G34, AGC 191.

Fig. 12. Camarozonotriletes sp. A A1-33, 8079-8082ft., Slide 715B, U37/4, AGC 192. 
J. B. Richardson

Plate 17

Emsian Miospores
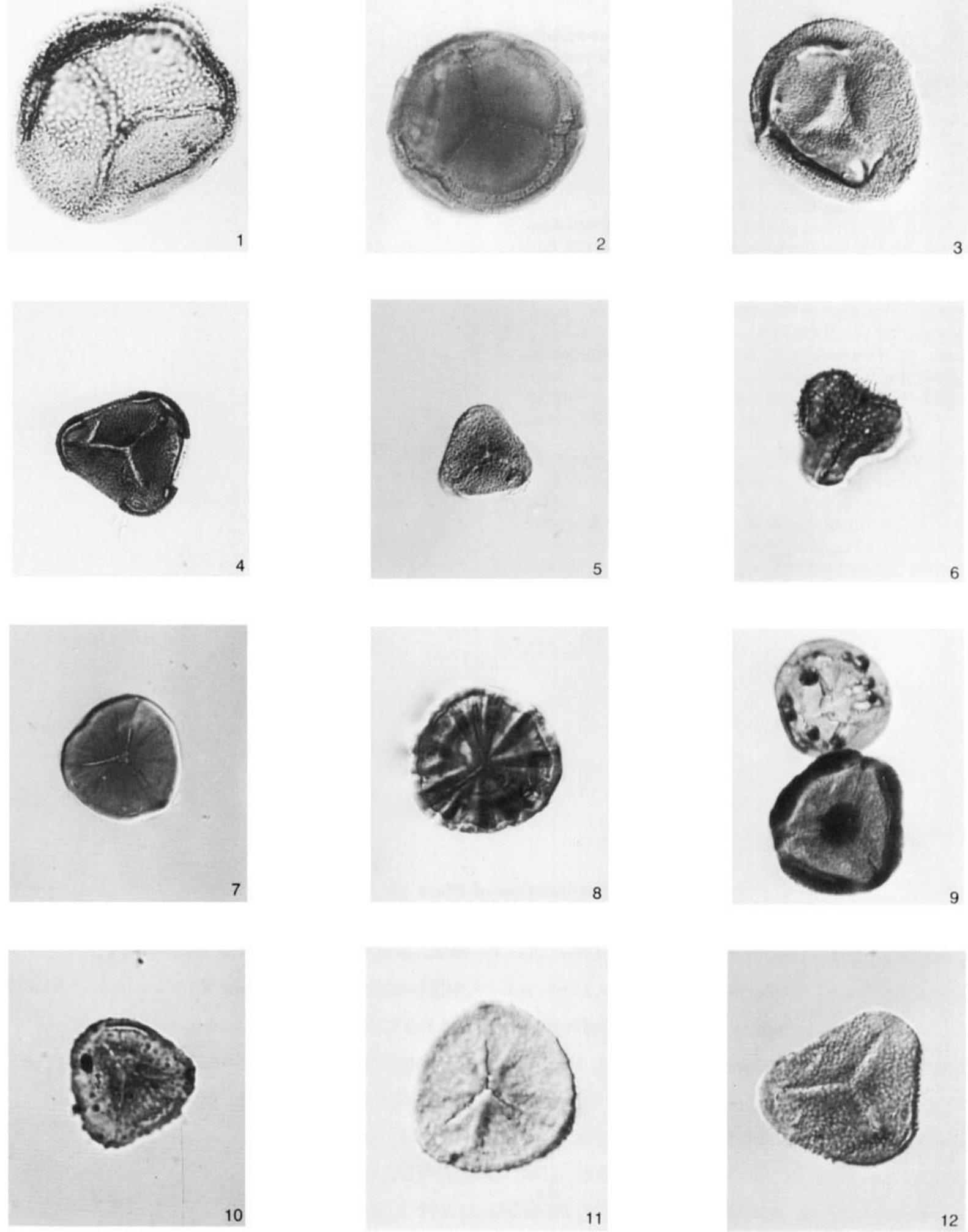
Massa, D. \& Moreau-Benoit, A. 1976. Essai de synthèse stratigraphique et palynologique du Système Devonien en Libye occidentale. Rev. Inst. Français Pétrole, 31, 287-332.

Playford, G. \& Dring, R. S. 1981. Late Devonian Acritarchs from the Carnarvon Basin, Western Australia. Special Papers in Paleontology, 27, 1-78.

Riegel, W. 1974. Spore floras across the Lower-Middle Devonian boundary in the Rhineland (G.R.F.). Palynology of the Proterophyte and Palaeophyte. Proc. 3rd Int. Palynol. Conf., 47-53.

Stockmans, F. \& Willière, Y. 1969. Acritarches du Famennien inferieur. Mem. Acad. roy. Belg. Cl. Sc., 38 (6), 1-63.

Stockmans, F. \& Willière, Y. 1974. Acritarches de la Tranchée de Senzeilles (Frasnien supérieur et Famennien inférieur) Mém. Acad. roy. Belg. Cl. Sc., 41 (5), 1-79.

Taugourdeau, P. 1965. Trois petites associations de Chitinozoaires du Frasnien du Boulonnais. Rev. Micropal., 8 (2), 6470.

Urban, J. B. \& Newport, R. L. 1973. Chitinozoa of the Wapsipinicon Formation (M. Devonian) of Iowa. Micropaleontology, 19 (2), 239-246.

Vanguestaine, M., Declairfayt, T., Rouhart, A. \& Smeesters, A. 1983. Zonation par Acritarches du Frasnien Supérieur Famennien Inférieur dans les bassins de Dinant, Namur, Herve et Campine (Dévonien supérieur de Belgique). Ann. Soc. Géol. Belg., 106, 121-171.

Wicander, E. R. 1974. Upper Devonian - Lower Mississippian Acritarchs and Prasinophycean algae from Ohio, U.S.A. Palaeontographica, B 148 (1-3), 9-43.

Wicander, E. R. \& Loeblich, A. R. Jr. 1977. Organic-walled microphytoplankton and its stratigraphic significance from the Upper Devonian Antrim Shale, Indiana, U.S.A.Palaeontographica, B 160 (4-6), 129-165.

\section{Explanation of Plate 18}

All figures are $\times 1000$

Fig. 1. Emphanisporites obscurus McGregor, 1961. A1-33, 8082-8085ft., Slide 716A, P28, AGC 193.

Fig. 2. Emphanisporites cf. obscurus McGregor, 1961. A1-33, 8082-8085ft., Slide 716A, K42/4 - L43/1, AGC 194.

Fig. 3. Emphanisporites erraticus (Eisenack) McGregor, 1961. A1-33, 8082-8085ft., Slide 716A, F43/1, AGC 195.

Fig. 4. Emphanisporites ?annulatus McGregor, 1961. A1-33, 8088-8091 ft., Slide 718A, M35/1, AGC 196.

Fig. 5. Emphanisporites cf. decoratus Allen, 1965. A1-33, 8082-8085ft., Slide 716A, M33/1, AGC 197.

Fig. 6. Aneurospora sp. A. A1-33, 8082-8085ft., Slide 716A, K33, AGC 198.

Fig. 7. Aneurospora sp. B. A1-33, 8082-8085ft., Slide 716A, F32/2, AGC 199.

Fig. 8. Cymbosporites sp. A1-33, 8082-8085ft., Slide 716A, S41/2, AGC 200.

Fig. 9. Craspedispora sp. A1-33, 8079-8082ft., Slide 715A, T36/4, AGC 201. 
J. B. Richardson Emsian Miospores
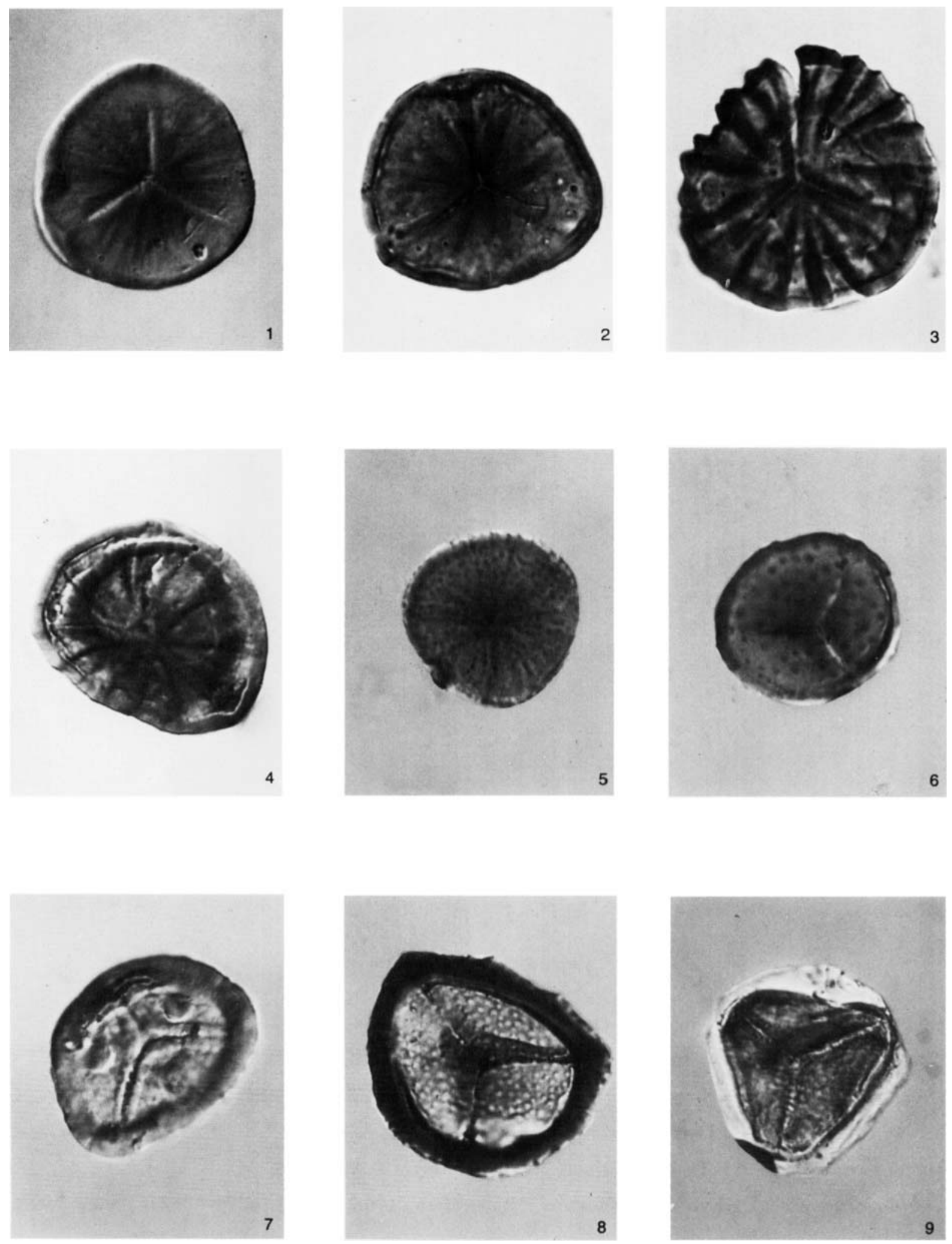

Plate 18 


\section{Explanation of Plate 19}

All figures are $\times 1000$

Fig. 1. ?Dibolisporites sp. B. A1-33, 8064-8067ft., Slide 711A, Q30/4, AGC 202.

Fig. 2. Diatomozonotriletes sp. A. (contaminant), A1-33, 8088-8091ft., Slide 718B, K40/3, AGC 203.

Fig. 3. Dibolisporites eifeliensis (Lanninger) McGregor, 1973. A1-33, 8082-8085ft., Slide 716A, J43, AGC 204.

Fig. 4. Procoronaspora sp. A1-33, 8082-8085ft., Slide 716A, N32, AGC 205.

Fig. 5. Dibolisporites sp. C. A1-33, 8079-8082ft., Slide 715A, Q40, AGC 206.

Fig. 6. Dibolisporites sp. cf. gibberosus (Naumova) Richardson, 1965. A1-33, 8079-8082ft., Slide 715A, L43, AGC 207. 
J. B. Richardson

Plate 19

Emsian Miospores
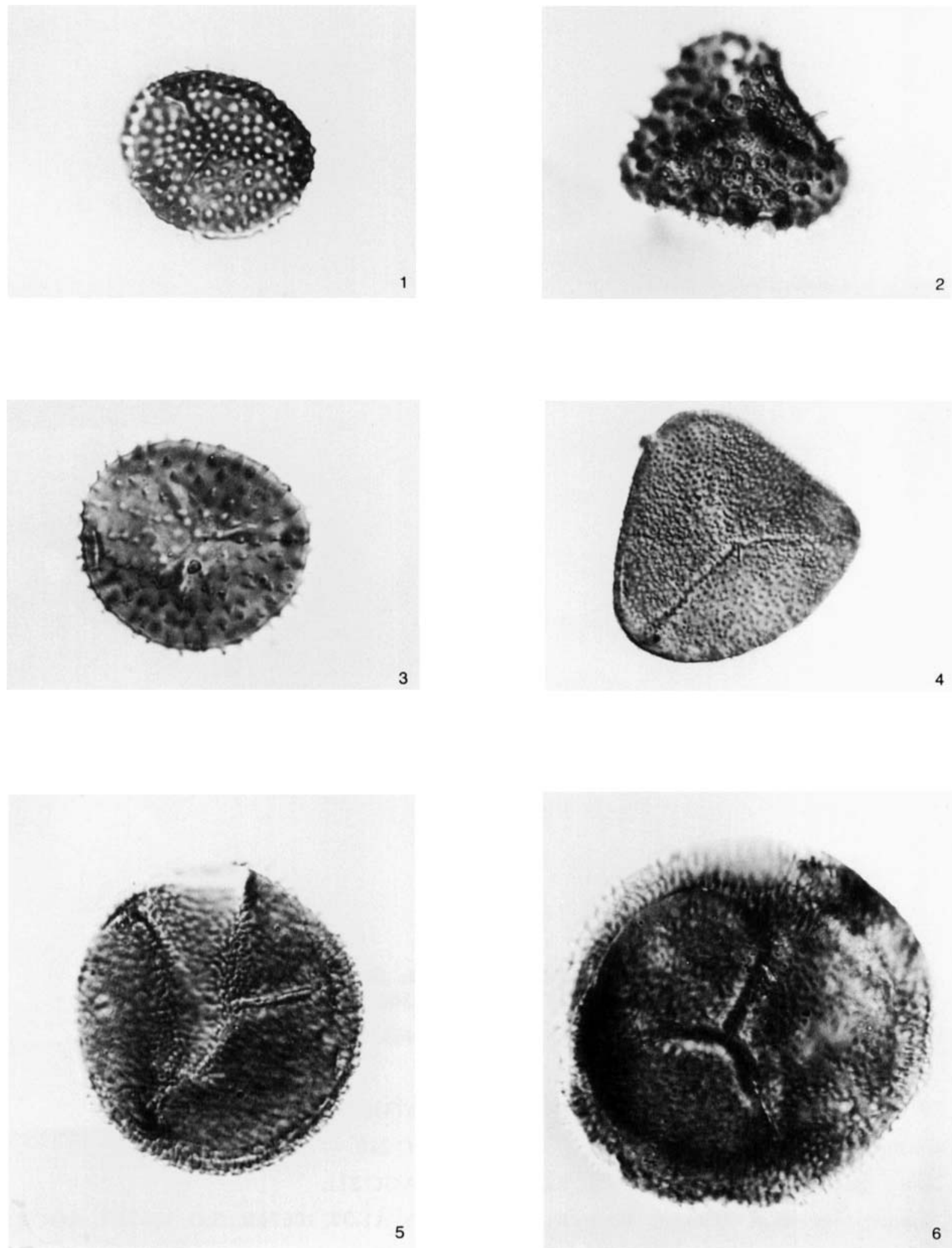


\section{Explanation of Plate 20}

All figures are $\times 500$

Fig. 1-2. cf. "Hymenozonotriletes" biformis Archangelskaya, 1963. A1-37, 10674ft., S.G. 10674/14, AGC 208.

Fig. 3. Zonate sp. A. A1-37, 10674 ft., Slide 3, AGC 269.

Fig. 4. "Hymenozonotriletes" discors (Chibrickova) A1-37, 10674ft., S.G. 10674/5, AGC 209.

Fig. 5. Craspedispora sp. A1-37, $10674 \mathrm{ft}$., S.G. 10674/20, AGC 210.

Fig. 6. Samarisporites sp. B. A1-37, 10674ft., S.G. 10674/16, AGC 211.

Fig. 7. ?Spinozonotriletes cf. naumovii (Kedo) Richardson, 1965. A1-37, 10674ft., S.G. 10674/1, AGC 212.

Fig. 8. ?Calyptosporites sp. A. A1-37, 10674 ft., S.G. 10674/8, AGC 213. 
J. B. Richardson

Plate 20

\section{Eifelian Miospores}
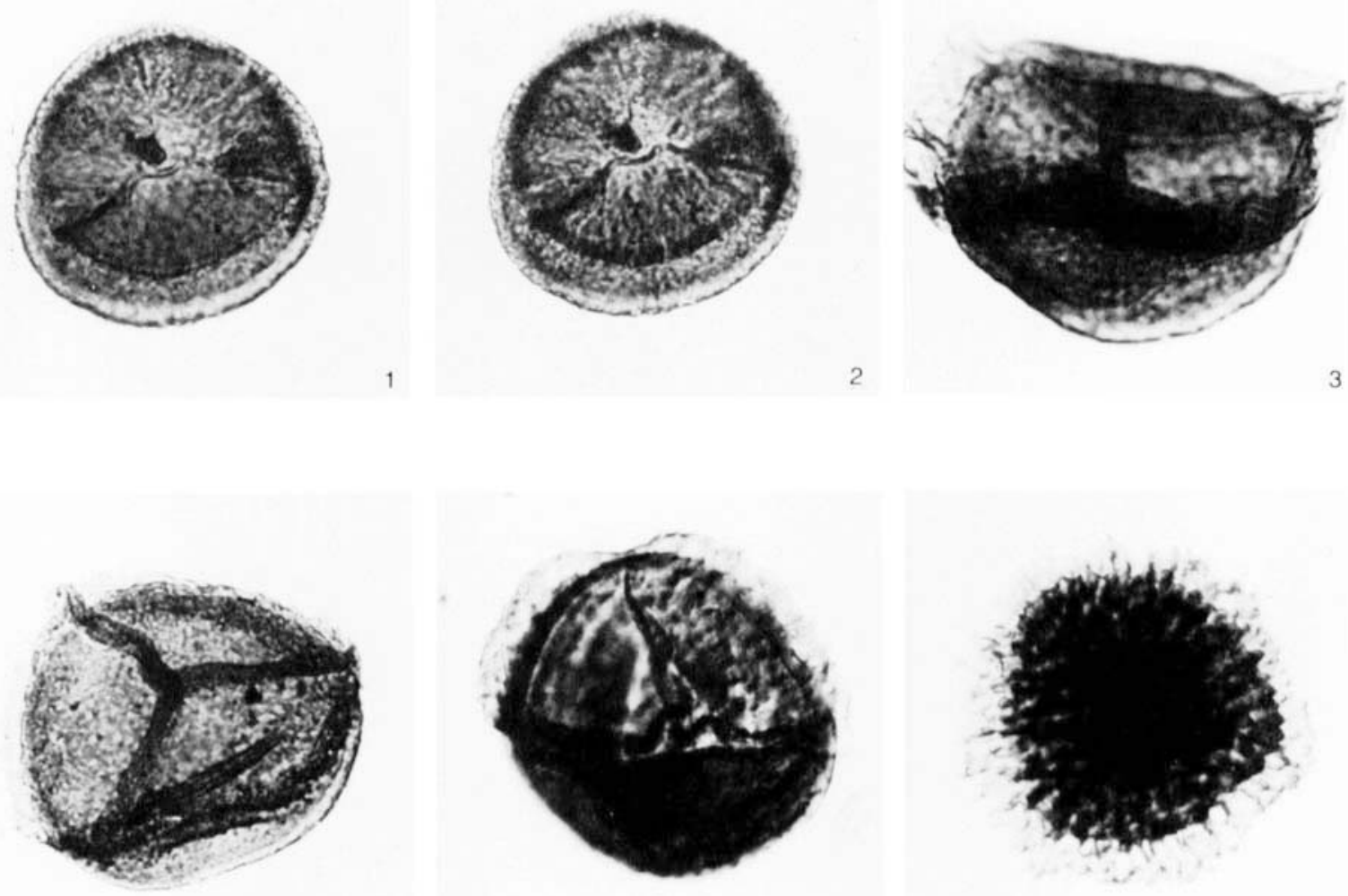

4

5
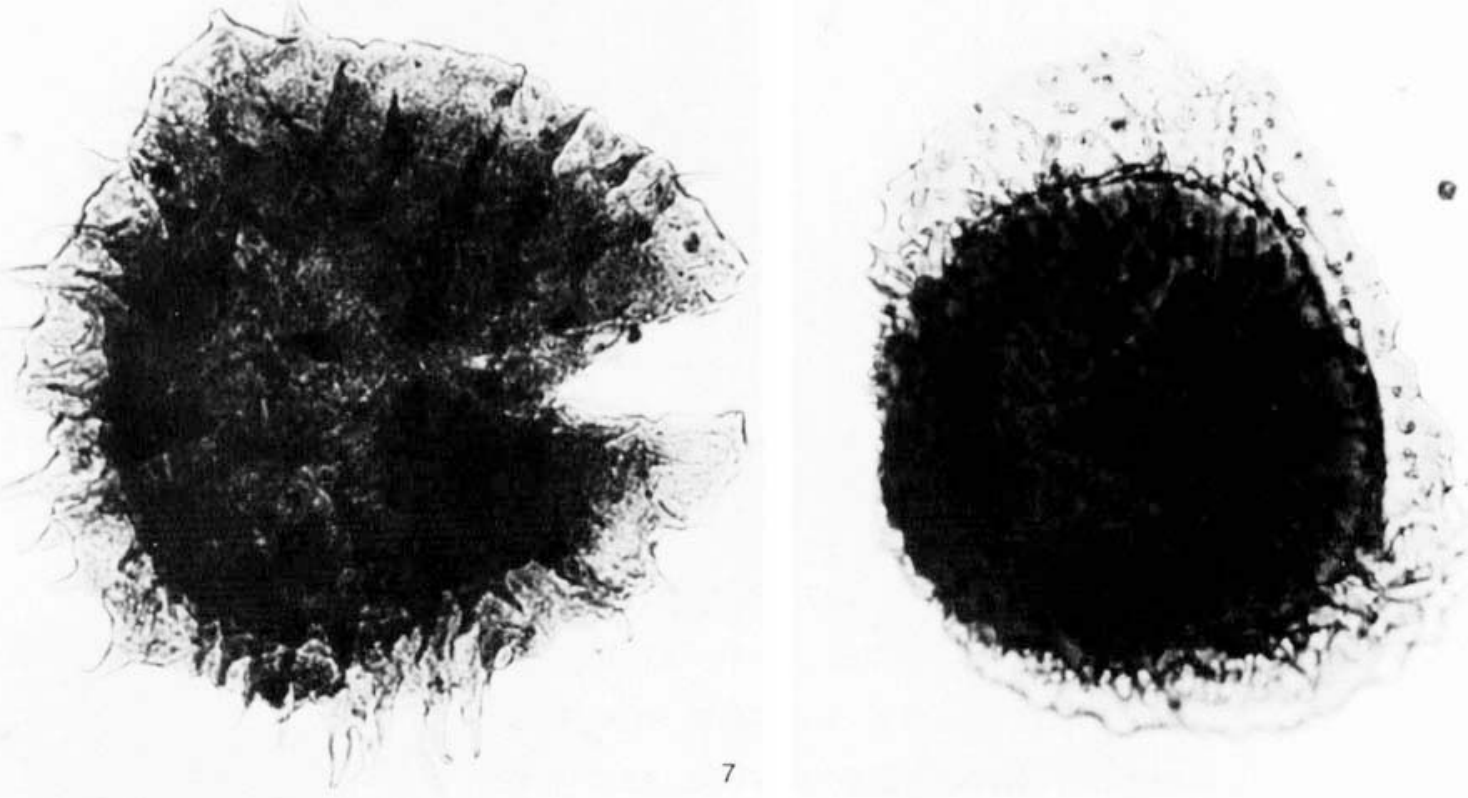
Fig. 1. ?Calyptosporites sp. A. A1-37, 10674ft., S.G. 10674/15, AGC 214.

Fig. 2-3. Samarisporites sp. A. A1-37, 10674 ft., S.G. 10674/23, AGC 215.

Fig. 4. cf. "Hymenozonotriletes" discors Chibrickova, 1959. A1-37, 10674ft., S.G. 10674/21, AGC 216.

Fig. 5. ?Contagisporites sp. A1-37, 10674ft., S.G. 10674/10, AGC 217.

Fig. 6. Calyptosporites sp. B. A1-37, 10674ft., S.G. 10674/2, AGC 218.

Fig. 7. Calyptosporites cf. velatus (Eisenack) Richardson, 1962. A1-37, 10674ft., S.G. 10674/6, AGC 219. 
J. B. Richardson

Plate 21

Eifelian Miospores
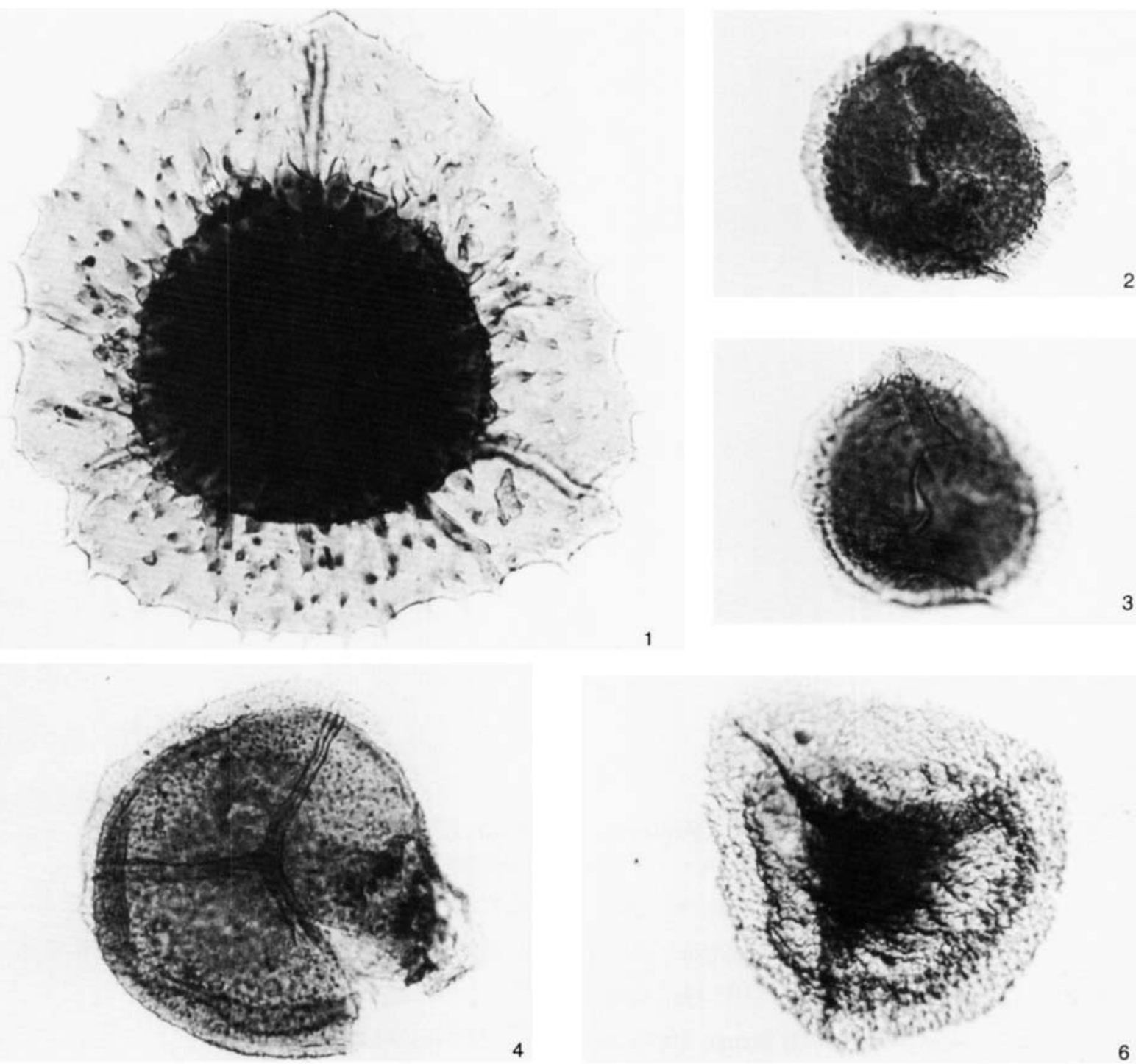

4
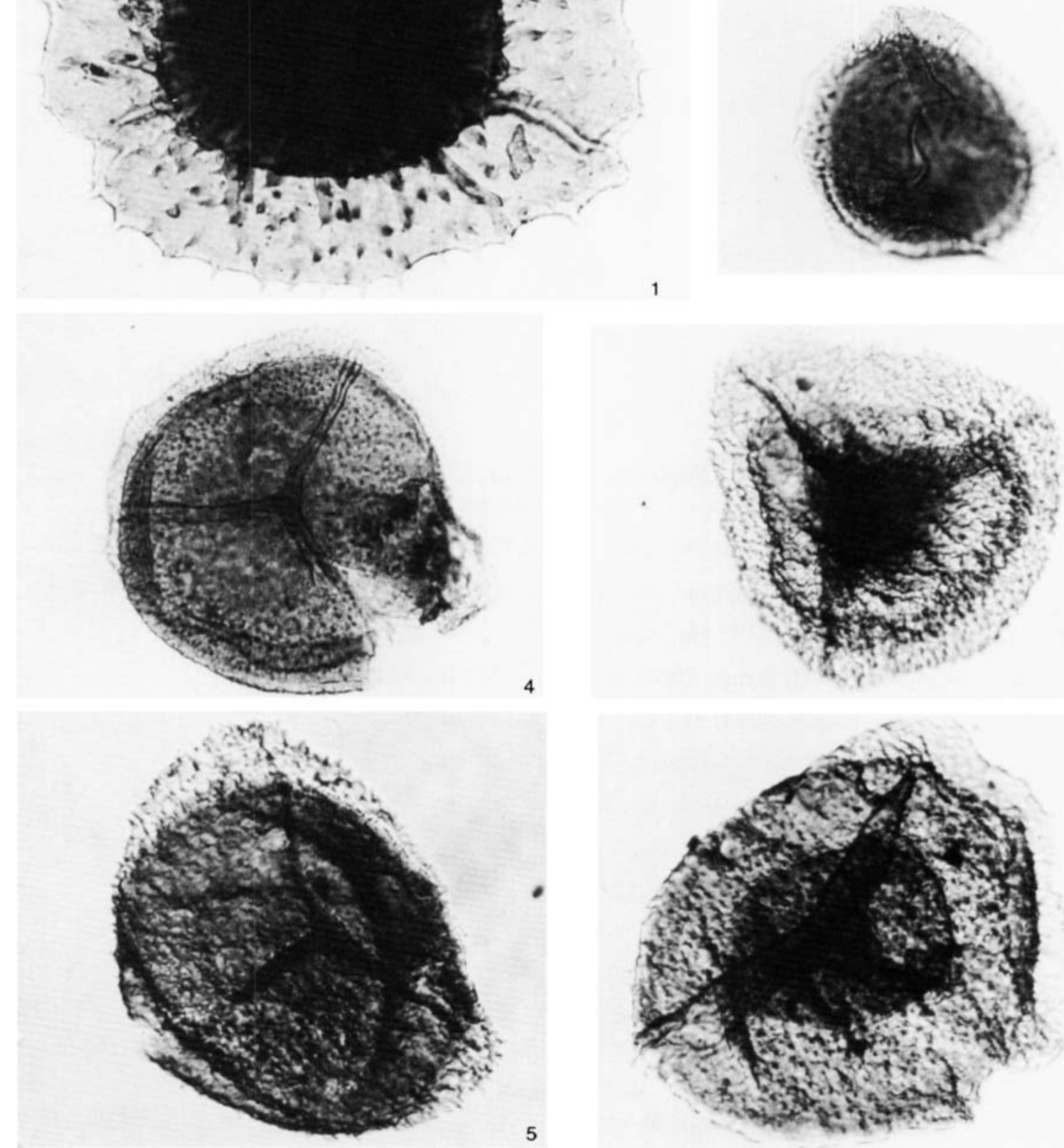


\section{Explanation of Plate 22}

All figures are $\times 500$ unless otherwise indicated

Fig. 1. Retusotriletes actinomorphus Chibrikova, 1962. E1-82, Core 7, 1319m., S.G. 1319/36, AGC 220.

Fig. 2. Retusotriletes triangulatus (Streel), Streel 1967. E1-82, Core 7, 1319m., S.G. 1319/19, AGC 221.

Fig. 3. Retusotriletes sp. E1-82, Core 7, 1319 m., S.G. 1319/56, AGC 222.

Fig. 4. Retusotriletes rotundus (Streel) Streel, 1967. J1-81A, $15685 \mathrm{ft.}$., AGC 223.

Fig. 5. Retusotriletes rugulatus Riegel, 1973. J1-81 A, $15685 \mathrm{ft}$., AGC 224.

Fig. 6. Punctatisporites sp. E1-82, Core 7, 1319m., S.G. 1319/44, AGC 226.

Figs. 7, 8. Cyclogranisporites sp. E1-82, Core 7, 1319m., S.G. 1319/15, AGC 225.

Fig. 9. Apiculiretusispora plicata (Allen) Streel, 1967. E1-82, Core 7, 1319m., S.G. 1319/9, AGC 227.

Fig. 10. Apiculiretusispora ef. brandtii (Streel). E1-82, Core 7, 1319m., S.G. 1319/1, AGC 228.

Fig. 11. Apiculiretusispora arenorugosa McGregor, 1973. J1-81 A, $15685 \mathrm{ft}$., AGC 229.

Figs. 12, 13. Apiculiretusispora brandtii Streel, 1964. E1-82, Core 7, 1319m., S.G. 1319/20, (Fig. 13, Detail of specimen in Fig. 12, $1000 \times$ ), AGC 230.

Fig. 14. Apiculiretusispora cf. brandtii Streel, 1964. E1-82, Core 7, 1319 m., S.G. 1319/68, AGC 231. Specimen with widely detached outer layer.

Fig. 15. Dibolisporites cf. gibberosus (Naumova) var. major (Kedo) Richardson, 1965. J1-81 A, $15685 \mathrm{ft}$., AGC 232.

Fig. 16-17. Verrucosisporites sp. E1-82, Core 7, 1319m., S.G. 1319/2, AGC 233. 

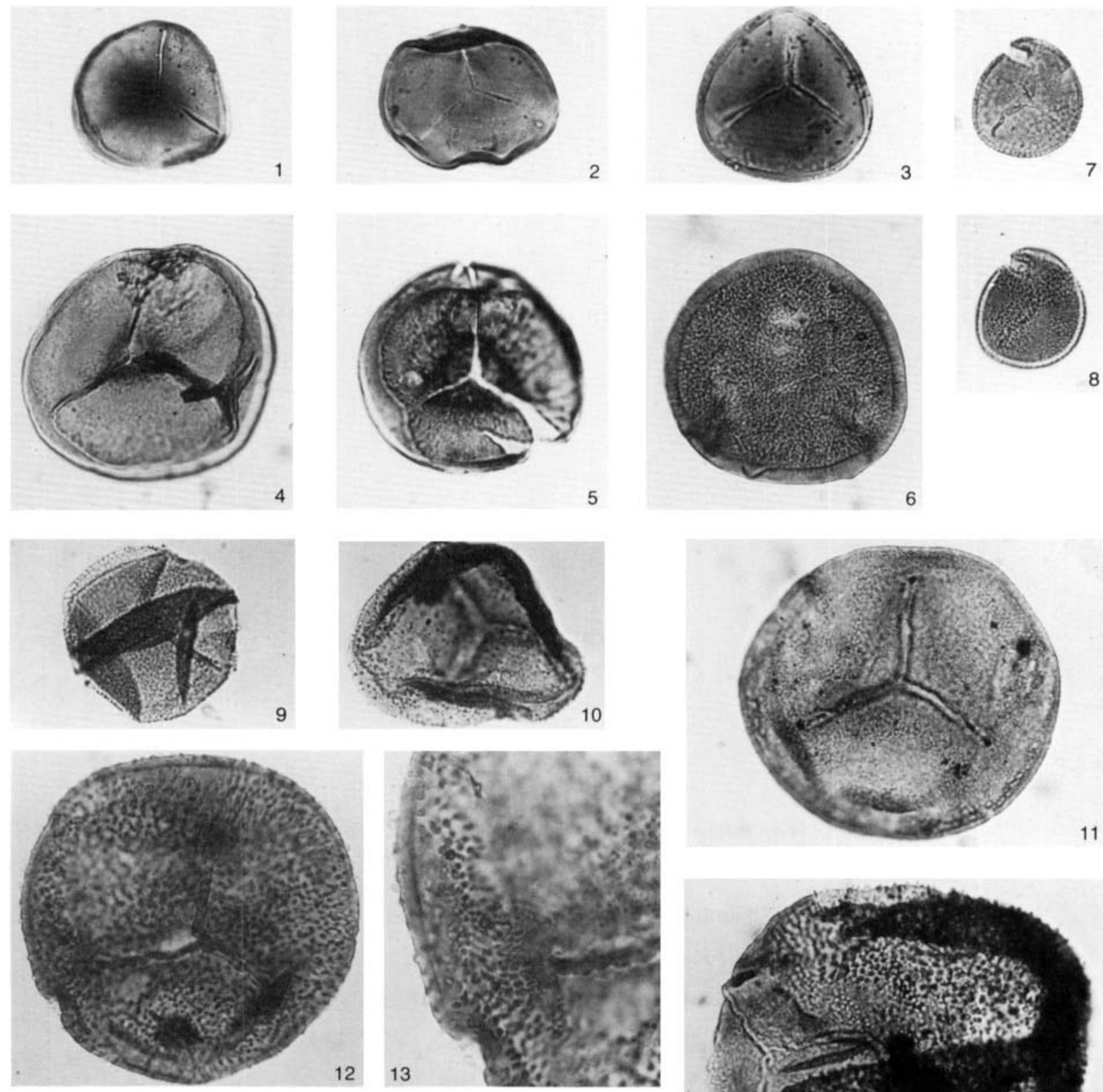

11

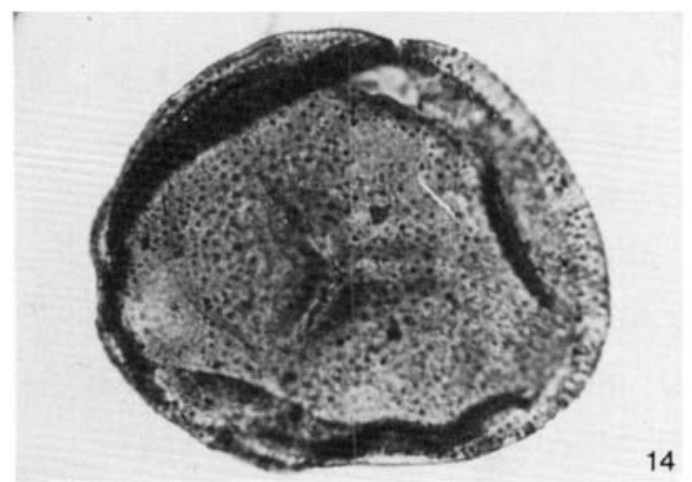

14
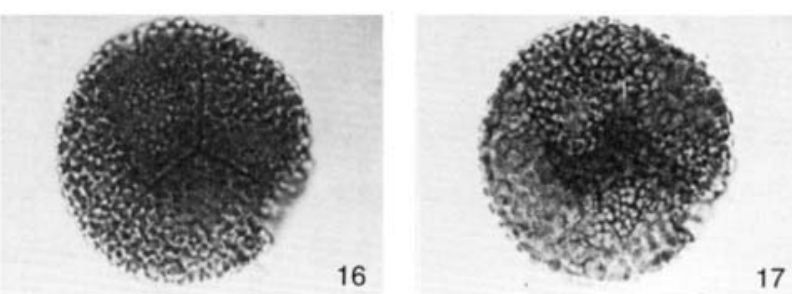


\section{Explanation of Plate 23}

All figures are $\times 500$

Fig. 1. Dibolisporites echinaceus (Eisenack) Richardson, 1965. E1-82, Core 7, 1319m., S.G. 1319/7, AGC 234.

Figs. 2, 3. Raistrickia aratra Allen, 1965. E1-82, Core 7, 1319m., S.G. 1319/26, AGC 235.

Figs. 4, 5. Verrucosisporites scurrus (Naumova) McGregor \& Camfield, 1982. J1-81A, $15685 \mathrm{ft}$., AGC 236.

Fig. 6. Raistrickia cf. aratra Allen, 1965. J1-81A, $15685 \mathrm{ft}$., AGC 237.

Fig. 7. Verrucosisporites cf. premnus Richardson, 1965. J1-81A, $15685 \mathrm{ft}$., AGC 238.

Fig. 8. Lophozonotriletes sp. E1-82, Core 7, 1319m., S.G. 1319/62, AGC 239.

Fig. 9. Verrucosisporites scurrus (Naumova) McGregor \& Camfield, 1982. E1-82, Core 7, 1319 m., S.G. 1319/80, AGC 240.

Fig. 10. Lophotriletes sp. E1-82, Core 7, 1319 m., S.G. 1319/58, AGC 241.

Fig. 11. Dictyotriletes sp. E1-82, Core 7, 1319m., S.G. 1319/4, AGC 242.

Fig. 12. Convolutispora sp. E1-82, Core 7, 1319m., S.G. 1319/27, AGC 243.

Fig. 13. Brochotriletes sp. E1-82, Core 7, 1319m., S.G. 1319/8, AGC 244.

Fig. 14. Dictyotriletes sp. E1-82, Core 7, 1319m., S.G. 1319/79, AGC 245.

Fig. 15. Acinosporites cf. apiculatus (Streel) Streel, 1964. E1-82, Core 7, 1319m., S.G. 1319/32, AGC 246.

Figs. 16, 17. Acinosporites sp. E1-82, Core 7, 1319m., S.G. 1319/53, AGC 848.

Fig. 18. Acinosporites acanthomammillatus Richardson, 1965. E1-82, Core 7, 1319m., S.G. 1319/57, AGC 247. 
W. Riegel

Plate 23

Givetian Miospores
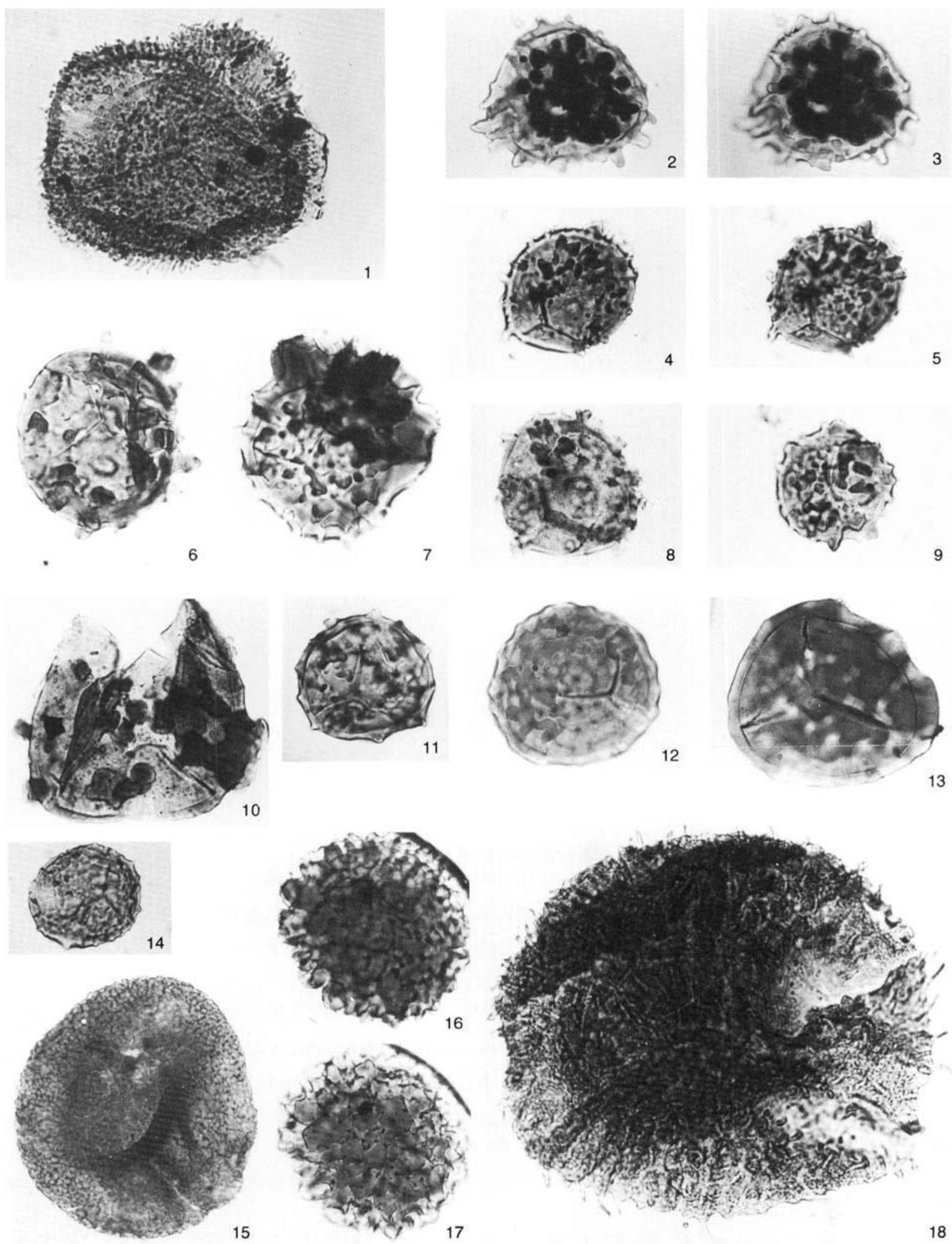


\section{Explanation of Plate 24}

\section{All figures are $\times 500$ unless otherwise indicated}

Figs. 1, 2. Emphanisporites spinaeformis Schultz, 1968. E1-82, Core 7, 1319m., S.G. 1319/10, AGC 248.

Fig. 3. Emphanisporites sp. with faint proximal radial ribs and narrow thin zona. E1-82, Core 7, 1319 m., S.G. 1319/ 30, AGC 249.

Fig. 4. Anapiculatisporites sp. E1-82, Core 7, 1319m., S.G. 1319/14, AGC 250.

Figs. 5, 6. Diatomozonotriletes sp. E1-82, Core 7, 1319m., S.G. 1319/16, AGX 251.

Fig. 7. Grandispora libyensis Moreau-Benoit, 1980. E1-82, Core 7, 1319m., S.G. 1319/44, AGC 252.

Figs. 8, 9. Grandispora sp. A. E1-82, Core 7, 1319 m., S.G. 1319/12, AGC 253. (Fig. 9: Detail of specimen in fig. 8). Compare with Daemon et al. 1967, pl. 3, fig. 35, 36 and Bär \& Riegel 1974, pl. 1, fig. 13).

Fig. 10. Grandispora sp. B. E1-82, Core 7, 1319 m., S.G. 1319/25, AGC 254. Finely sculptured form with cingulate central body.

Figs. 11, 12, 13. Grandispora sp. C. E1-82, Core 7, 1319m., S.G. 1319/18, AGC 255. (Fig. 12, 13: Details of specimen in fig. $11,1000 \times$ ). 
W. Riegel

Plate 24

Givetian Miospores
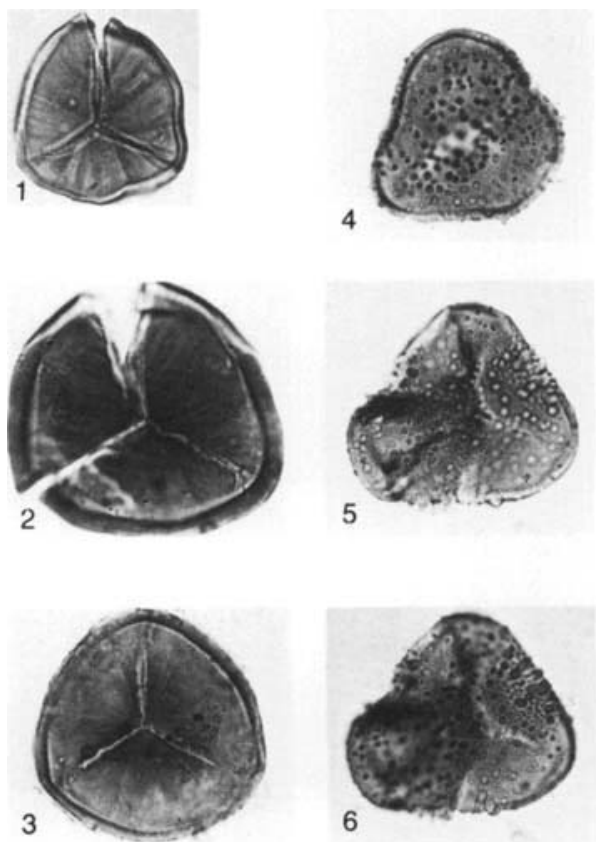
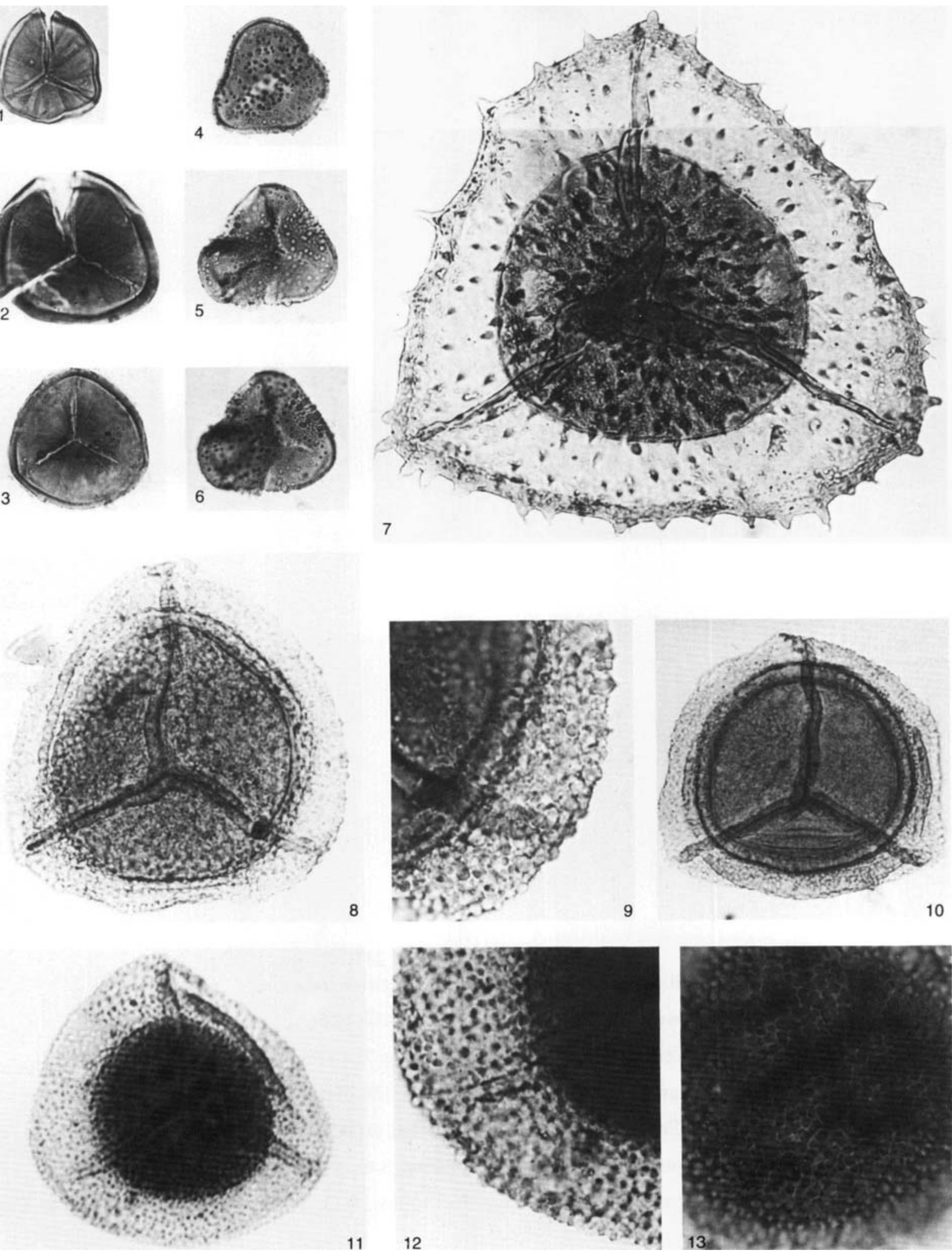


\section{Explanation of Plate 25}

All figures are $\times 500$ unless otherwise indicated

Fig. 1. Geminospora sp. E1-82, Core 7, 1319 m., S.G. 1319/76, AGC 256.

Fig. 2. Geminospora tuberculata (Kedo) Allen, 1965. E1-82, Core 7, 1319m., S.G. 1319/28, AGC 257.

Fig. 3. Calyptosporites proteus (Naumova) Allen, 1965. J1-81 A, 15685ft., AGC 258.

Fig. 4. Rhabdosporites sp. E1-82, Core 7, 1319m., S.G. 1319/64, AGC 259.

Figs. 5, 6. "Monolete" verrucate spore. E1-82. Core 7, 1319 m., S.G. 1319/2, AGC 260.

Figs. 7, 8. Hystricosporites sp. megaspore. E1-82. Core 7, 1319m., S.G. 1319/11, AGC 261. (Fig. 8: Detail of specimen in Fig. 7, 1000 $\times$ ).

Fig. 9. Biharisporites sp. E1-82. Core 7, 1319 m., S.G. 1319/40, AGC 262. 


\section{Givetian Miospores}
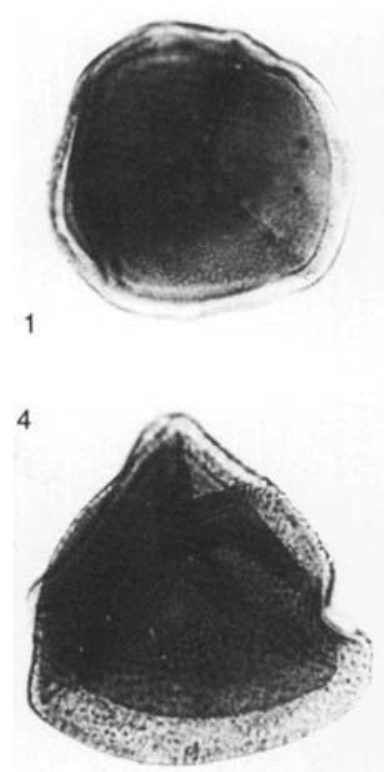

8
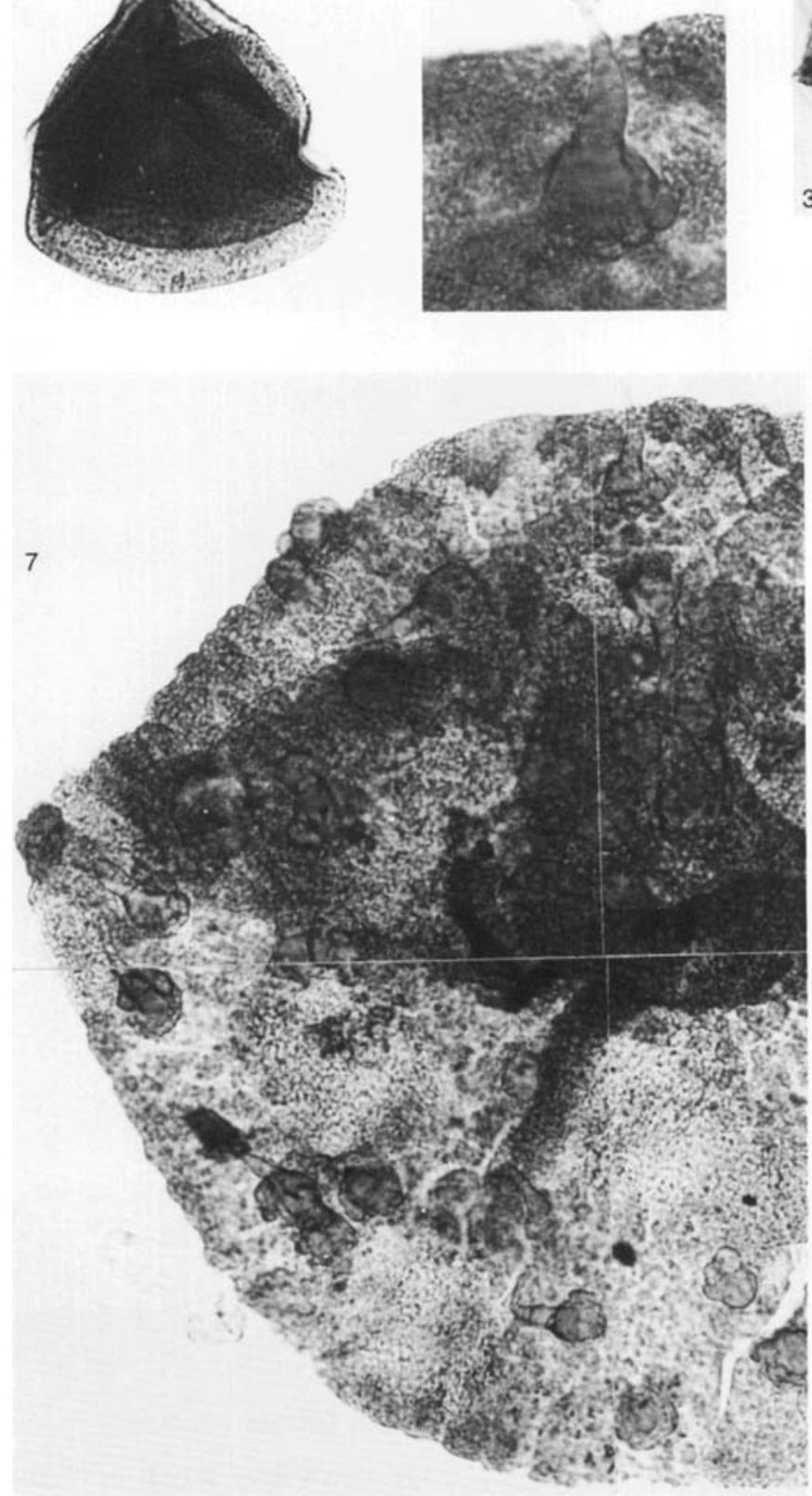

2

3
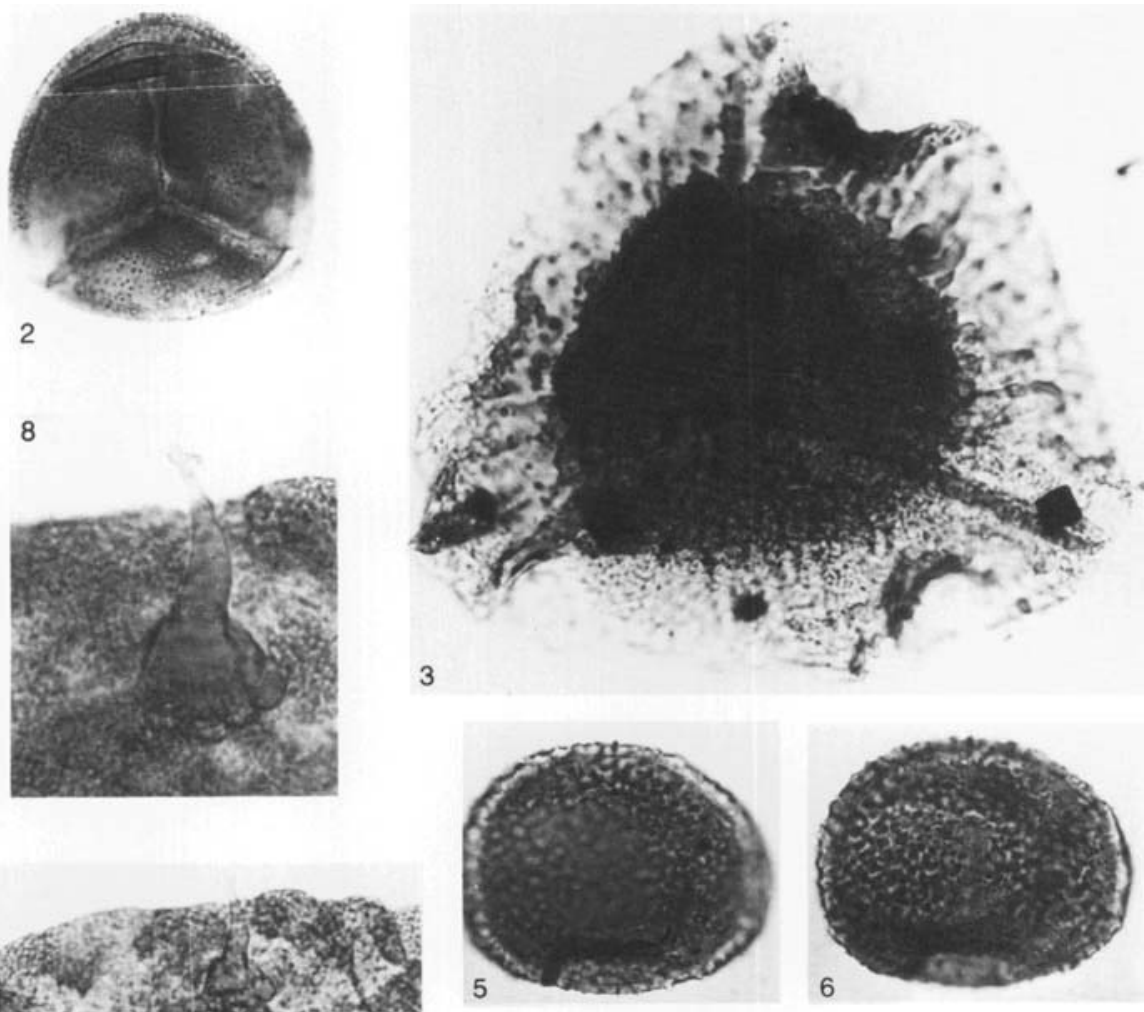

9

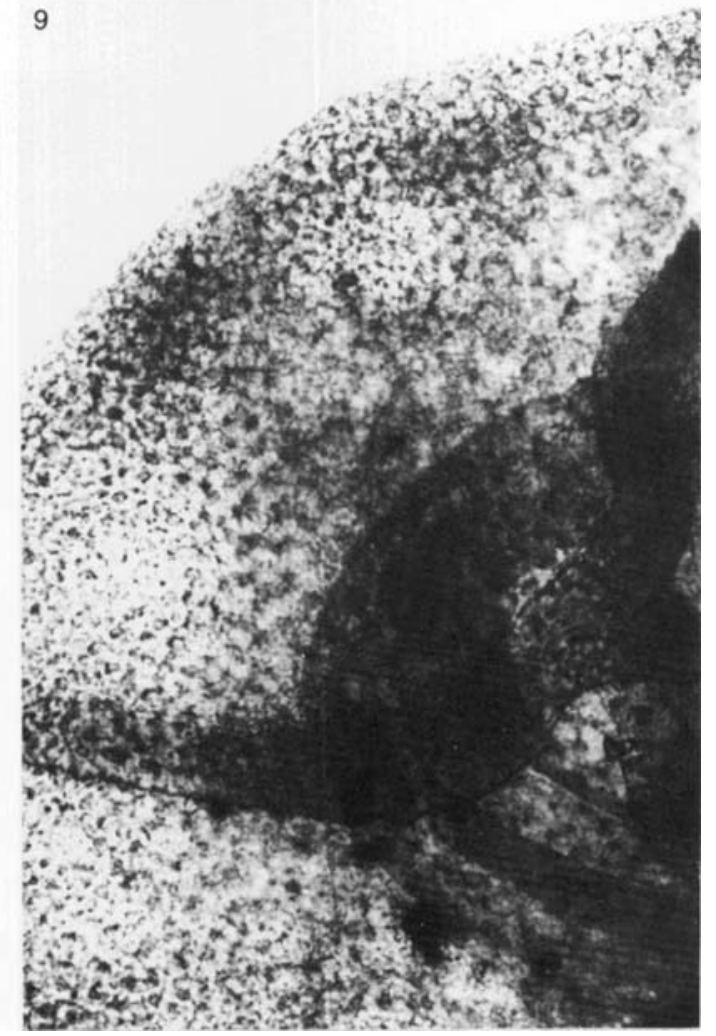


Fig. 1. Tasmanites sp. 11C-81, SWC 5806, AGC 263.

Fig. 2. Leiosphaeridia sp. 11C-81, SWC 5806, AGC 264.

Fig. 3. Gorgonisphaeridium sp. I1C-81, SWC 5806, AGC 265.

Fig. 4. Polyedryxium pharaonis Deunff, 1961. J1-81A, $15685 \mathrm{ft} .$, AGC 266.

Figs. 5, 6. Polyedryxium fragosulum Playford, 1977. I1C-81, SWC 5806, AGC 267. (phase contrast).

Fig. 7. Incertae sedis. E1-82, Core 7, 1319m., S.G. 1319/40, AGC 268. Bipolar body with 4 resp. 6 hollow "bifurcate" appendages at poles (see Doubinger 1966). 
W. Riegel

Givetian Phytoplankton
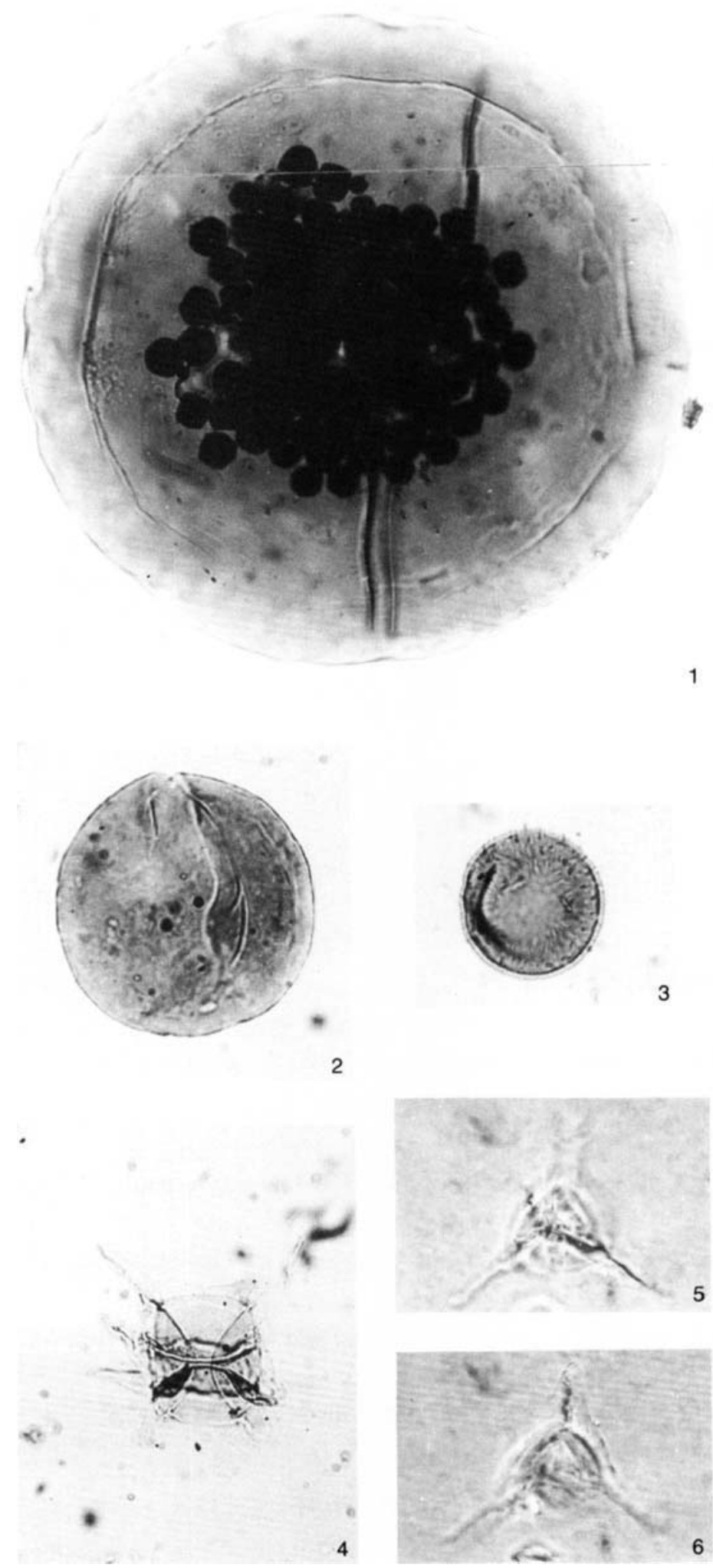

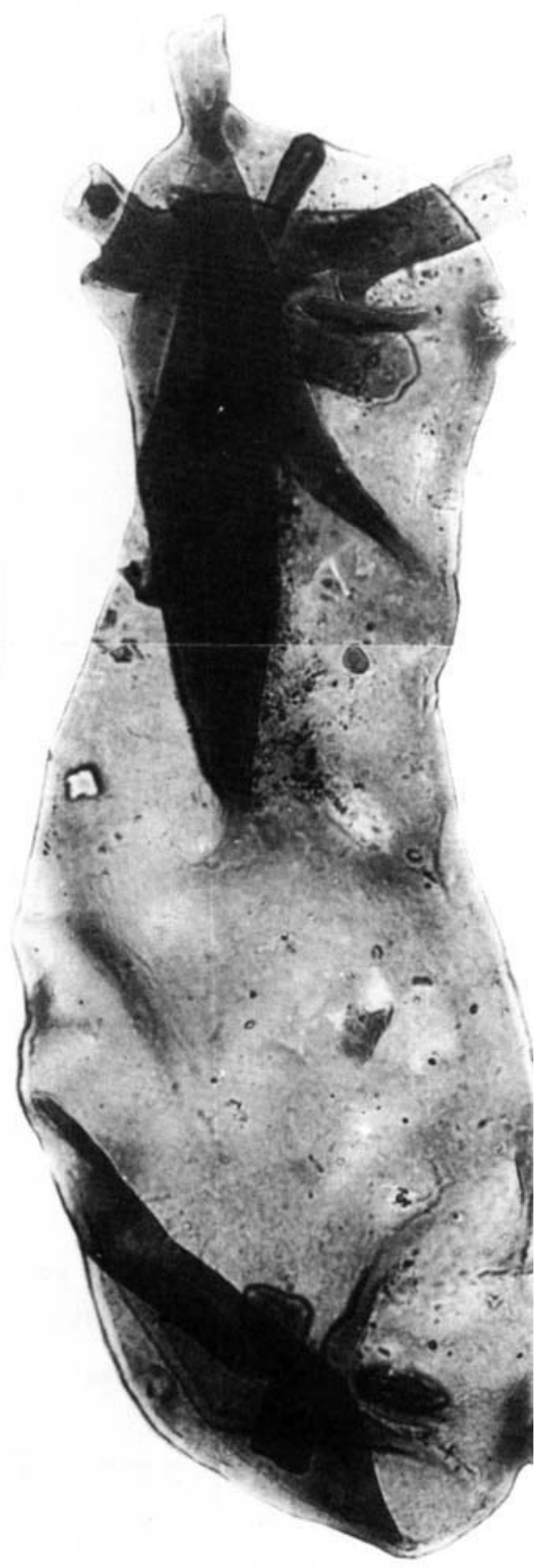




\section{Explantion of Plate 27}

Fig. 1a, b. Eisenackitina castor Jansonius, 1964. sensu Legault, 1973. A1-37, $9840 \mathrm{ft}$., Slide 13, N35/2, 1a: $\times 300 ; 1$ b: $\times 750$, AGC 270. (The spinous forms are restricted to the Givetian while E. castor s.s. occurs in Late Eifelian).

Fig. 2a, b. Eisenackitina castor Jansonius, 1964. sensu Legault, 1973. A1-37, $9840 \mathrm{ft}$., Slide 13, Q35/1, 2a: $\times 300 ; 2 b$ : $\times 1250$, AGC 271. (Givetian).

Fig. 3. Oral pole (collarette and operculum) of Eisenackitina castor Jansonius, 1964, sensu Legault, 1973. A1-37, $9840 \mathrm{ft}$., Slide 13,038/2, × 750, AGC 272.

Fig. 4. Eisenackitina castor Jansonius, 1964, sensu Legault, 1973. A1-37, 9840ft., Slide 13, Q37/4, × 300, AGC 273. (Givetian).

Fig. 5. Eisenackitina castor Jansonius, 1964, sensu Legault, 1973. A1-37, 9840ft., Slide 13, M34/4, × 400, AGC 274. (Givetian).

Fig. 6a, b. Gotlandochitina? sp. A. A1-37, $9840 \mathrm{ft}$., Slide 13, N38, 6a: $\times 300 ; 6 \mathrm{~b}: \times 750$, AGC 275.

Fig. 7. Gotlandochitina sp. A1-37, 9840ft., Slide 13, P36/4, × 400, AGC 276.

Fig. 8. Gotlandochitina milanensis (Collinson \& Scott, 1958). A1-37, 9840ft., Slide 13, P38/4, ×300, AGC 277. (Givetian).

Fig. 9. Ancyrochitina ? aequoris Urban \& Kline, 1970. A1-37, $9840 \mathrm{ft}$., Slide 13, 037/3, ×300, AGC 278. (Givetian). 

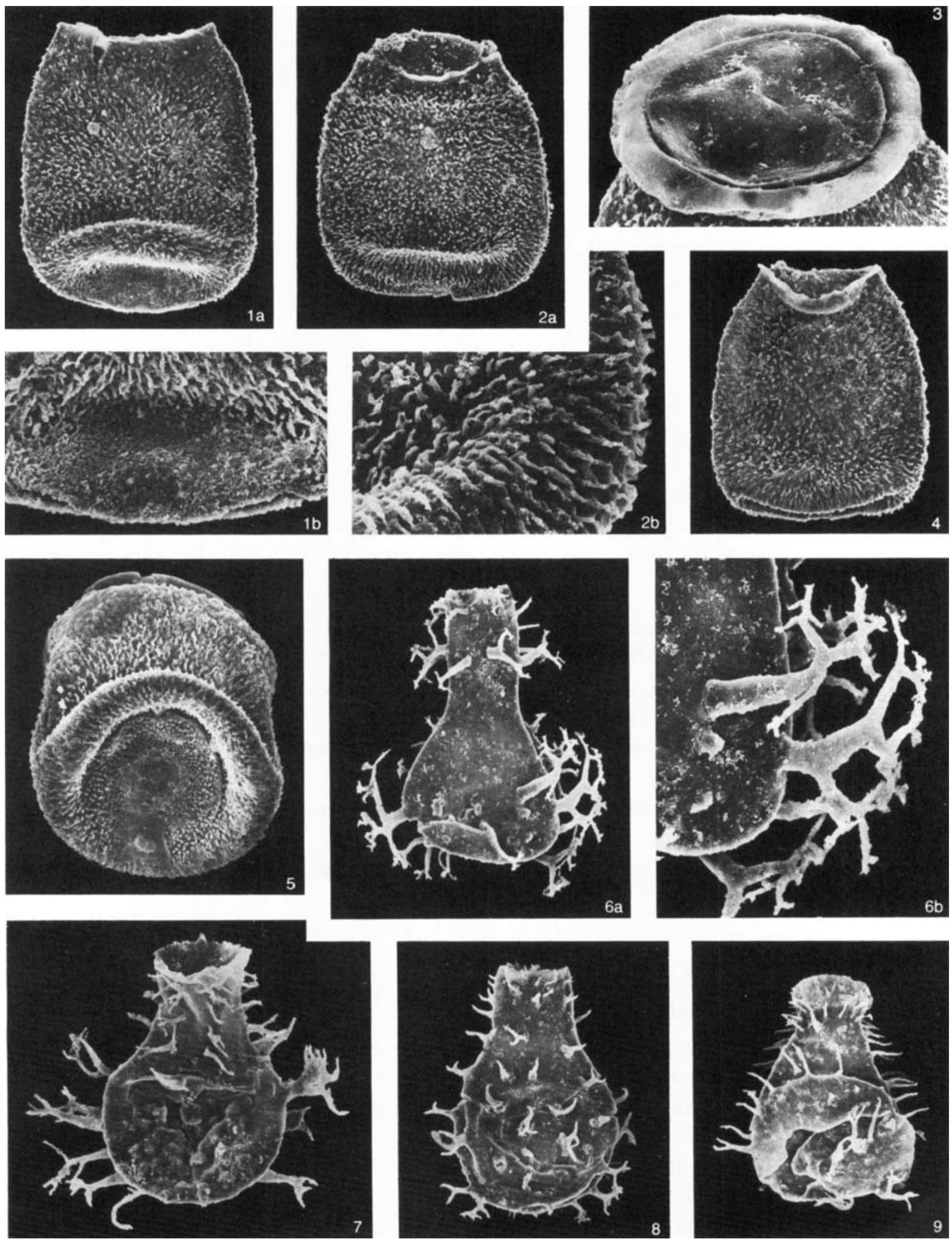


\section{Explanation of Plate 28}

Fig. 1a, b. Alpenachitina eisenacki Dunn \& Miller, 1964. Contaminated cuttings from J1-81 A, 12800-12850ft., (Ashgill), Slide 2, N38/3, 1a: ×300; 1b: $\times 1000$, AGC 279. (Eifelian-Givetian).

Fig. 2. Alpenachitina eisenacki Dunn \& Miller, 1964. Contaminated cuttings from J1-81A, 12950-13000ft., (Ashgill), Slide 1, M39/4, ×300, AGC 280. (Eifelian-Givetian).

Fig. 3. Fungochitina pilosa (Collinson \& Scott, 1958). A1-37, $9251 \mathrm{ft}$., Slide 15, 039, $\times 300$, AGC 281. (EifelianFrasnian).

Fig. 4a, b. Fungochitina pilosa (Collinson \& Scott, 1958). A1-37, 9251 ft., Slide 15, 038, 4a: $\times 250 ; 4 \mathrm{~b}: \times 1250$, AGC 282. (Eifelian-Frasnian).

Fig. 5. Fungochitina pilosa (Collinson \& Scott, 1958). A1-37, $9251 \mathrm{ft}$., Slide 15, R43/1, × 400, AGC 283. (EifelianFrasnian).

Fig. 6. Fungochitina pilosa (Collinson \& Scott, 1958). A1-37, 9290ft., Slide 14, L43, × 300, AGC 284. (EifelianFrasnian).

Fig. 7a-b. Gotlandochitina sp. B. (= Angochitina devonica Eisenack, 1955, sensu Urban, 1972). A1-37, $9840 \mathrm{ft}$., Slide 13, Q35, 7a: $\times 250 ; 7 \mathrm{~b}: \times 1000$, AGC 285. (A. devonica s.l. is reported from the Pragian up to the Frasnian).

Fig. 8. Gotlandochitina sp. B. (=Angochitina devonica Eisenack, 1955, sensu Urban, 1972). A1-37, 9840ft., Slide 13, Q35/1, $\times 250$, AGC 286. (A. devonica s.l. is reported from the Pragian up to the Frasnian).

Fig. 9. Gotlandochitina sp. B. (=Angochitina devonica Eisenack, 1955, sensu Urban, 1972). A1-37, $9840 \mathrm{ft}$., Slide 13, P34, $\times 300$, AGC 287. (A. devonica s.l. is reported from the Pragian up to the Frasnian).

Fig. 10a, b. Fungochitina pilosa (Collinson \& Scott, 1958). A1-37, $9251 \mathrm{ft}$., Slide 15, Q37/2, 10a: $\times 300 ; 10 \mathrm{~b}: \times 1250$, AGC 288. (Eifelian-Frasnian).

Fig. 11 Hoegisphaera glabra Staplin, 1961. A1-37, 9840ft., Slide 13, Q34/1, × 300, AGC 289. (Late Pragian-Early Frasnian; typical $H$. glabra are restricted to the Early Frasnian). 
F. Paris

Plate 28

Givetian-Frasnian Chitinozoans
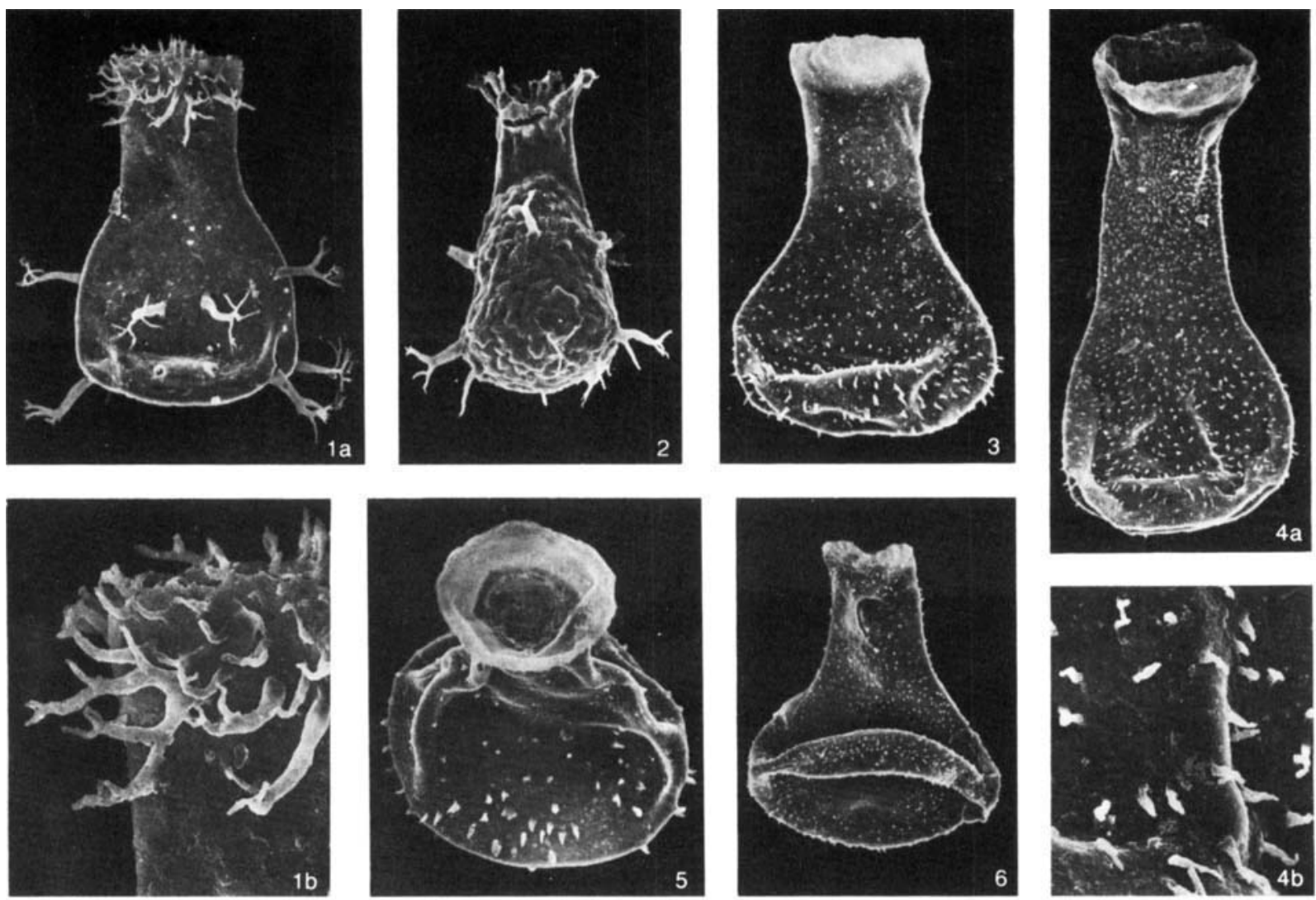

$4 a$
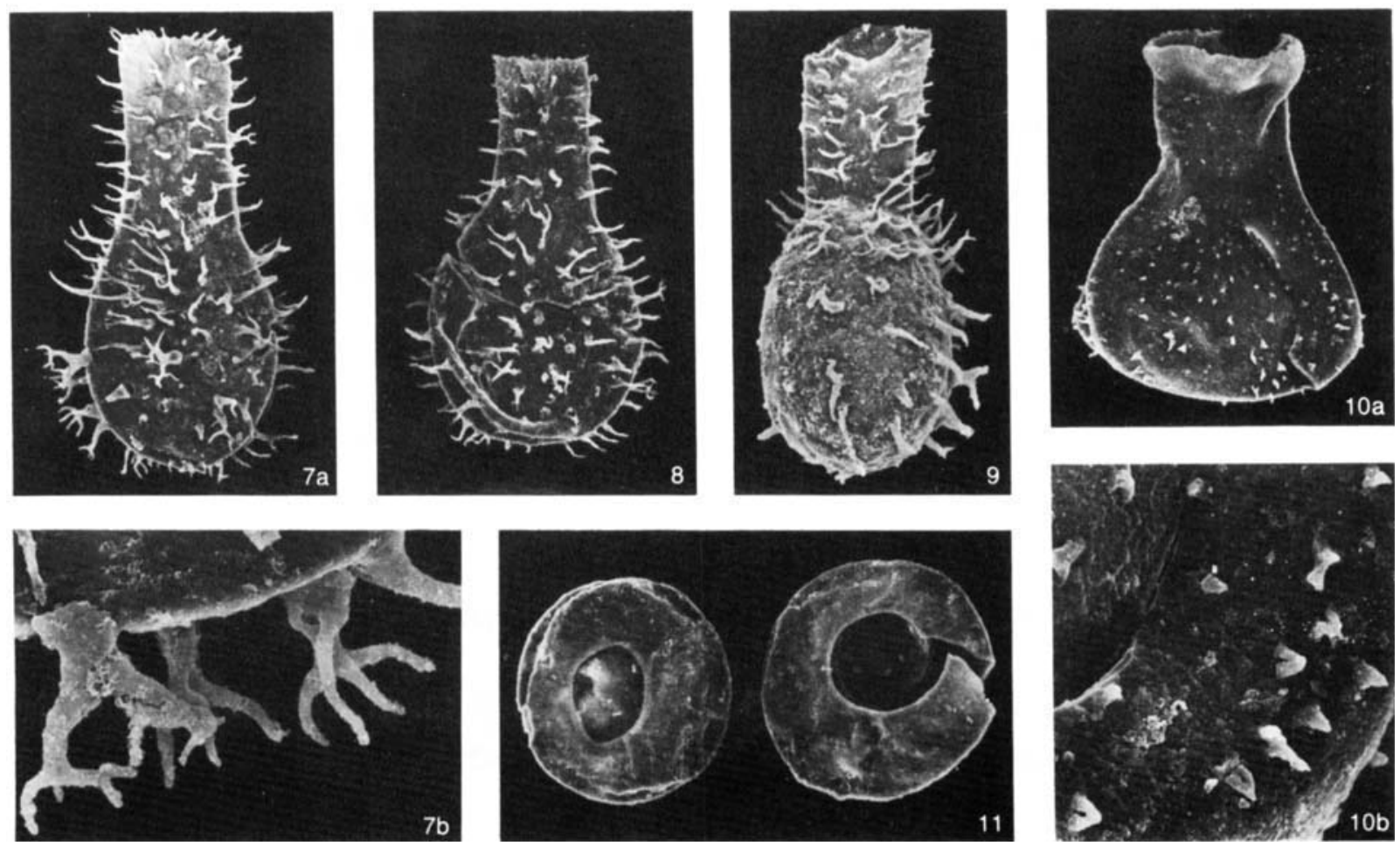


\section{Explanation of Plate 29}

All figures are $\times 500$

Fig. 1. Rugospora flexuosa (Juskho) Streel in Becker et al., 1974 A1-NC92, $13024 \mathrm{ft}$., Slide 1, R40, AGC 290 (Late Famennian).

Figs. 2, 3. Leiotriletes struniensis Moreau-Benoit, 1979. 2. A1-NC92, 13024ft., Slide 1, T41/1, AGC 291. 3. A1-NC92, $13024 \mathrm{ft} .$, Slide 1, Q40/1, AGC 292. (Late Famennian).

Fig. 4. Cyrtospora cristifer (Luber) Van der Zwan 1979. A1-NC92, 13400ft., Slide 1, H39/4, AGC293. (FamennianTournaisian).

Fig. 5. Geminospora lemurata Balme, 1962. A1-115, 8784 ft., S.G. 8784/3, AGC 294. (Givetian-Famennian).

Figs. 6, 7. Samarisporites triangulatus Allen, 1965. C1-125, 10700-10800ft., Slide 1, 044, AGC 295. 6. proximal view, 7. distal view. (Givetian-Frasnian).

Fig. 8. Verrucosisporites bullatus Taugourdeau-Lantz, 1967. C1-125, 10300-10400ft., Slide 1, F35/2, AGC 296. (Frasnian).

Figs. 9, 10. Verrucosisporites premnus Richardson, 1965. A1-37, 9638ft., S.G. 9638/47, AGC 297. 9. proximal view, 10. distal view. (Eifelian-Frasnian).

Fig. 11. Verrucosisporites scurrus McGregor \& Camfield, 1982. A1-37, 9638ft., S.G. 9638/20, AGC 298. (EifelianFrasnian).

Fig. 12. Rhabdosporites langi (Eisenack) Richardson, 1960. A1-37, 9638ft., S.G. 9638/44, AGC 299. (EifelianGivetian).

Fig. 13, 14. Samarisporites sp. A1-115, 8784ft., S.G. 8784/1, AGC 300. 13. distal view, 14. proximal view.

Figs. 15, 16. Acinosporites macrospinosus Richardson, 1965. A1-37, $9825 \mathrm{ft}$., S.G. 9825/12, AGC 301. (EifelianGivetian).

Fig. 17. Grandispora inculta Allen, 1965. A1-37, 9633 ft., S.G. 9633/17, AGC 302. (Givetian-Frasnian). 
M. Streel

Plate 29

Givetian-Famennian Miospores
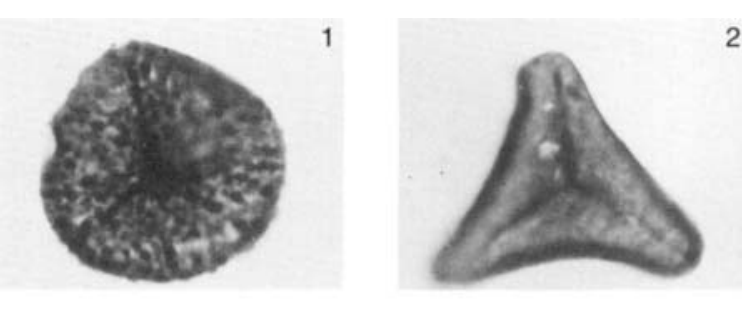

2
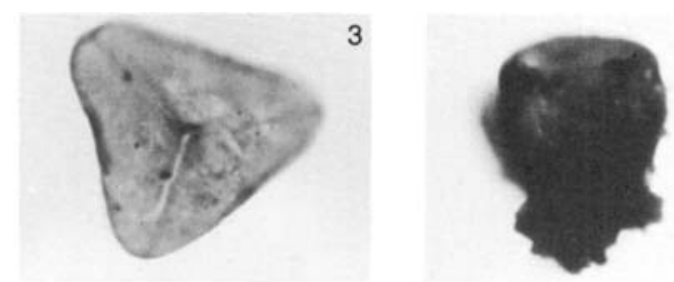

4
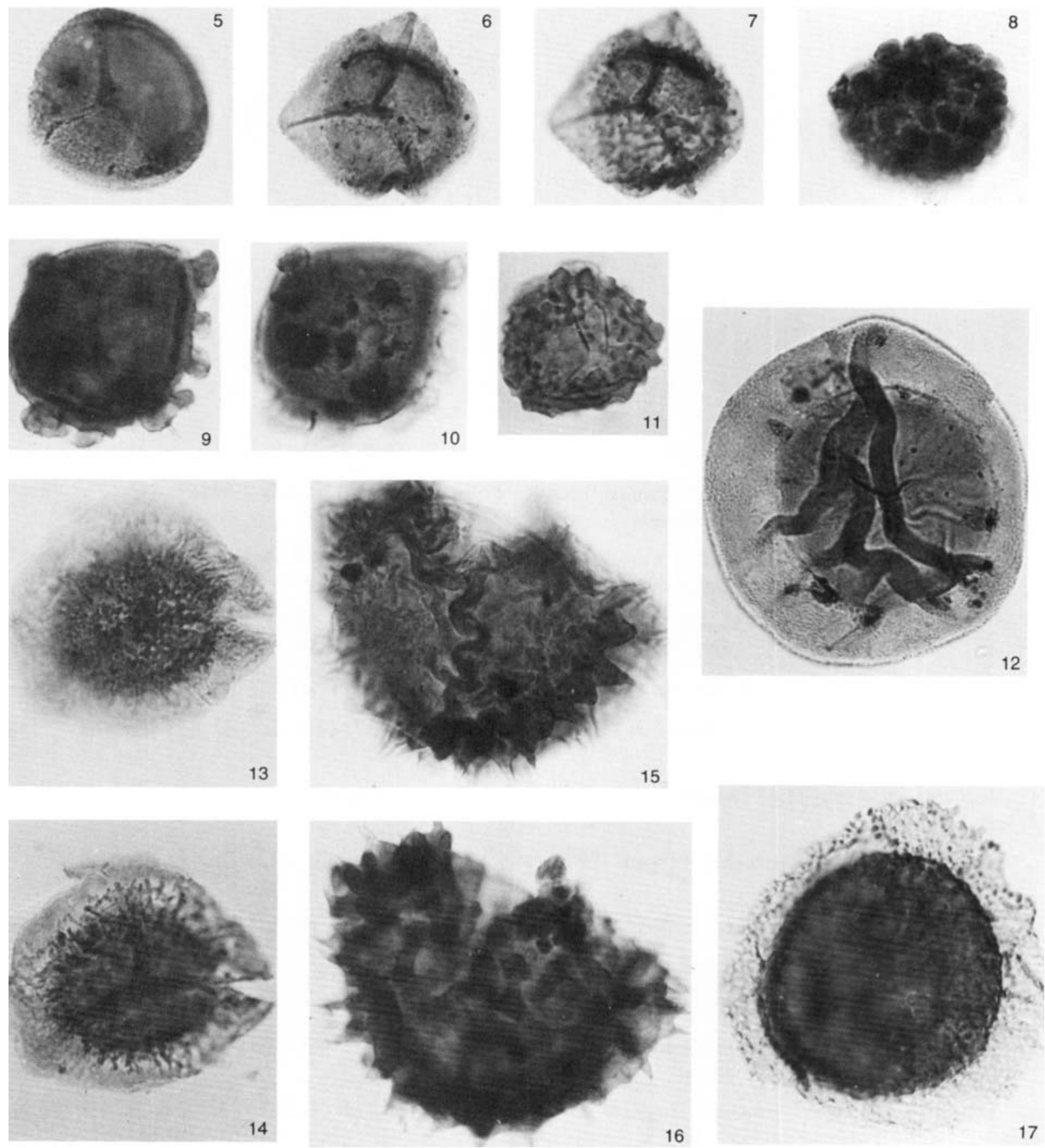


\section{Explanation of Plate 30}

All figures are $\times 500$

Fig. 1. Spelaeotriletes resolutus Higgs, 1975. A1-37, 8418ft., S.G. 8418/41, AGC 303. (Famennian-Tournaisian).

Fig. 2. Spelaeotriletes granulatus (Kedo) Moreau-Benoit, 1979. A1-37, 8421 ft., S.G. 8421/9, AGC 304 . Interference contrast view. (Famennian-Tournaisian).

Figs. 3, 4. Auroraspora asperella (Kedo) Van der Zwan, 1979. A1-37, 8418ft., S.G. 8418/7, AGC 305. 4. Interference contrast view. (Famennian-Tournaisian).

Figs. 5-7. Retispora lepidophyta (Kedo) Playford, 1976. 5. A1-37, 8421 ft., S.G. 8421/23, AGC 306. 6. A1-37, $8421 \mathrm{ft} .$, S.G. 8421/22, AGC 307. 7. A1-37, $8421 \mathrm{ft}$., S.G. 8421/18, AGC 308. (Late Famennian).

Fig. 8. Retusotriletes incohatus Sullivan, 1964. A1-37, 8418ft., S.G. 8418/11, AGC 309. (Famennian-Tournaisian).

Fig. 9. Rugospora flexuosa (Jushko) Streel in Becker et al., 1974. A1-37, 8418ft., S.G. 8418/14, AGC 310. (Late Famennian).

Fig. 10. Vallatisporites pusillites (Kedo) Dolby \& Neves, 1970. A1-37, 8418ft., S.G. 8418/6, AGC 311. (Late Famennian).

Fig. 11. Endoculeospora gradzinskii Turnau, 1975. A1-37, 8421ft., S.G. 8421/26, AGC 312. (FamennianTournaisian).

Fig. 12. Lophozonotriletes bellus Kedo, 1963. A1-37, 8418 ft., S.G. 8418/65, AGC 313. (Famennian-Tournaisian).

Fig. 13. Pustulatisporites sp. A1-37, 8418ft., S.G. 8418/38, AGC 314.

Fig. 14. Corbulispora sp. A1-37, 8418 ft., S.G. 8418/23, AGC 849.

Fig. 15. Knoxisporites literatus (Waltz) Playford, 1963. A1-37, 8418ft., S.G. 8418/5, AGC 315. (FamennianTournaisian).

Figs. 16-18. Spelaeotriletes cf. crustatus Higgs, 1975. 16, 17. A1-37, 8421 ft., S.G. 8421/37, AGC 316. 16. proximal view. 17. distal view. 18. A1-37, 8421 ft., S.G. 8421/21, AGC 317. (Famennian-Tournaisian). 
M. Streel

Plate 30

\section{Late Famennian Miospores}
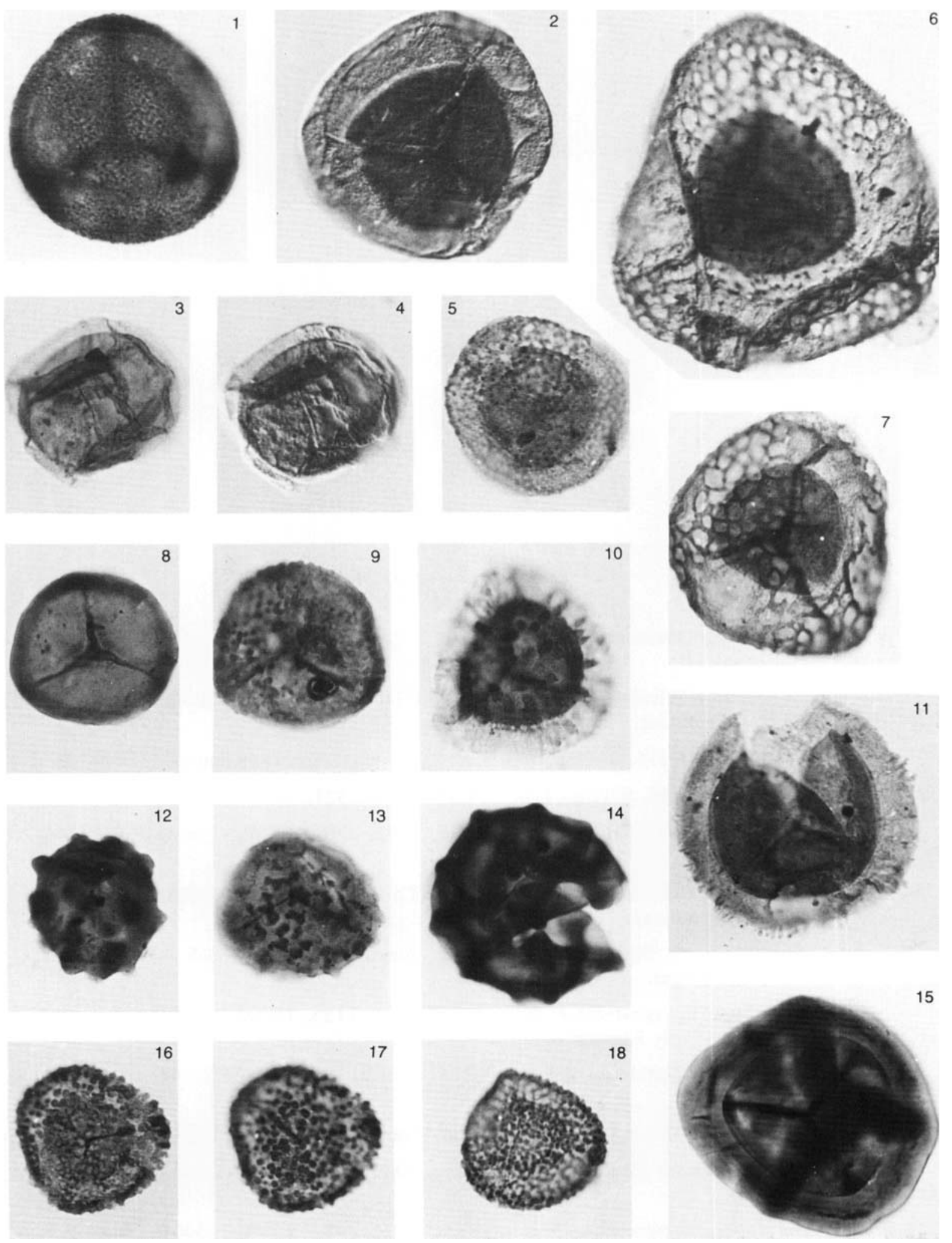


\section{Explanation of Plate 31}

All figures are $\times 1000$

Fig. 1. Veryhachium downiei Stockmans \& Willière, 1962. C1-125, 1149, 9800-9900ft., Slide 19500, J43, AGC 318. (Ordovician-Tournaisian).

Fig. 2. Veryhachium pannuceum Wicander \& Loeblich, 1977. C1-125, 1155, 10400-10500ft., Slide 19.523, H33/4, AGC 319. (Frasnian-Famennian).

Fig. 3. Veryhachium n. sp. A. A1-115, 8784ft., S.G. 8784/16A, AGC 320. (Famennian).

Fig. 4. Schizocystia n. sp. A. A1-115, 8772 ft., Slide 1, E25/4, AGC 321.

Fig. 5. Rugaletes cf. vietus Playford \& Dring, 1981. C1-125, 1152, 10100-10200ft., Slide 19520, J48, AGC 322. (Frasnian).

Fig. 6. Horologinella horologia (Staplin) Jardiné et al., 1972 C1-125, 1150, 9900-10000ft., Slide 19.500, S33/2, AGC 333. (Givetian-(Famennian).

Fig. 7. Stellinium micropolygonale (Stockmans \& Willière) Playford, 1977. A1-115, $8784 \mathrm{ft}$. , Slide 1, H33/1, AGC 334. (Emsian-Famennian).

Fig. 8. Villosacapsula globosa Vanguestaine et al., 1983. C1-125, 1155, 10400-10500ft., Slide 19.523, T33/4, AGC 335. (Late Frasnian-Early Famennian).

Fig. 9. Micrhystridium stellatum Deflandre, 1945. A1-115, $8784 \mathrm{ft}$., Slide 1, S38/2, AGC 336. (Silurian-Early Mesozoic).

Fig. 10. Tornacia stela Wicander, 1974. C1-125, 1153, 10200-10300ft., Slide 19.521, T44, AGC 337. (Famennian).

Fig. 11. Elektoriskos cf. deconinckii (Stockmans \& Willière, 1969) nov. comb. A1-115, 8784ft., Slide 1, J31/1, AGC 338. (Famennian).

Fig. 12. Villosacapsula globosa Vanguestaine et al., 1983. C1-125, 1156, 10500-10600ft., Slide 19.524, H39/4, AGC 339. (Late Frasnian-Early Famennian). 
M. Vanguestaine

Plate 31

Frasnian-Famennian Acritarchs
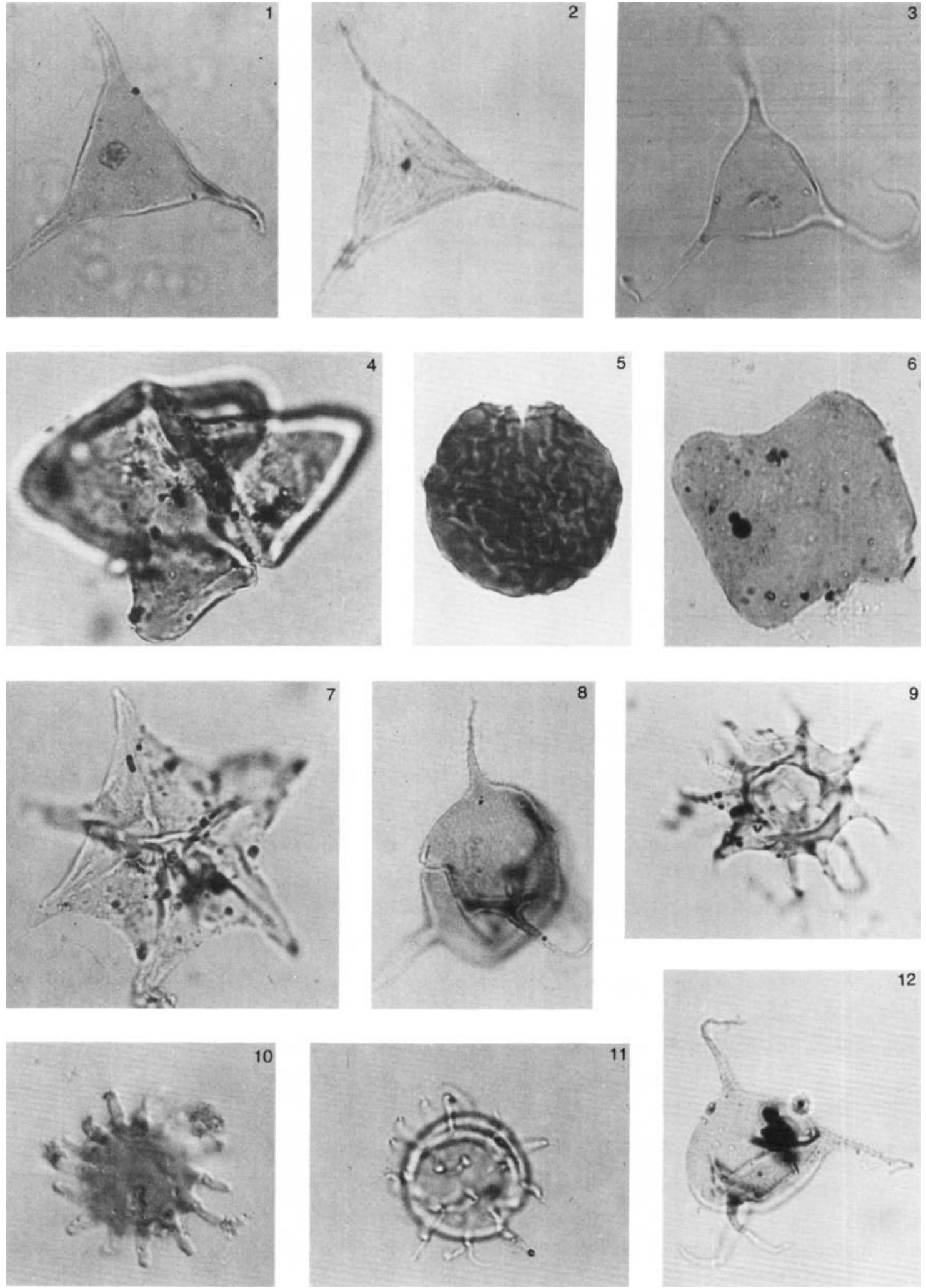


\section{Explanation of Plate 32}

All figures are $\times 1000$

Fig. 1. Gorgonisphaeridium n. sp. A. A1-115, 8784ft., S.G. 8784/3A, AGC 340.

Fig. 2. Unellium winslowae Rauscher, 1969. C1-125, 1150, 9900-10000ft., Slide 19500, F39, AGC 341 (FrasnianFamennian).

Fig. 3. Gorgonisphaeridium cf. ohioense (Winslow) Wicander, 1974. C1-125, 1150, 9900-10000ft., Slide 19500 , P45/2, AGC 342. (Famennian).

Fig. 4. Helosphaeridium n. sp. A. A1-115, 8774 ft., Slide 1, H30/4, AGC 343.

Fig. 5. Helosphaeridium n. sp. A. A1-115, 8784 ft., Slide 1, S42/2-S43/1, AGC 344.

Fig. 6. Chomotriletes aff. vedugensis Naumova, 1953 in Playford \& Dring, 1981. C1-125, 1150, 9900-10000ft., Slide 19500, J41, AGC 345.

Fig. 7. Gorgonisphaeridium n. sp. B. A1-115, 8772, Slide 1, Y41, AGC 346.

Fig. 8. Gorgonisphaeridium cf. ohioense (Winslow) Wicander, 1974. C1-125, 1150, 9900-10000ft., Slide 19500, Z38/3, AGC 347. (Famennian).

Fig. 9. Pirea sp. A. C1-125, 1150, 9900-10000ft., Slide 19500, M41/4, AGC 348.

Fig. 10. Lophosphaeridium cf. impensum Wicander \& Loeblich, 1977. A1-115, 8774ft., Slide 1, L46, AGC 349. (Famennian).

Fig. 11. Solisphaeridium apodasmion (Wicander) Wicander \& Loeblich, 1977. A1-115, 8784ft., Slide 1, R33/3, AGC 350. (Famennian).

Fig. 12. Gorgonisphaeridium sp. C. A1-115, 8772 ft., Slide 1, 037/4, AGC 351. 
Frasnian-Famennian Acritarchs
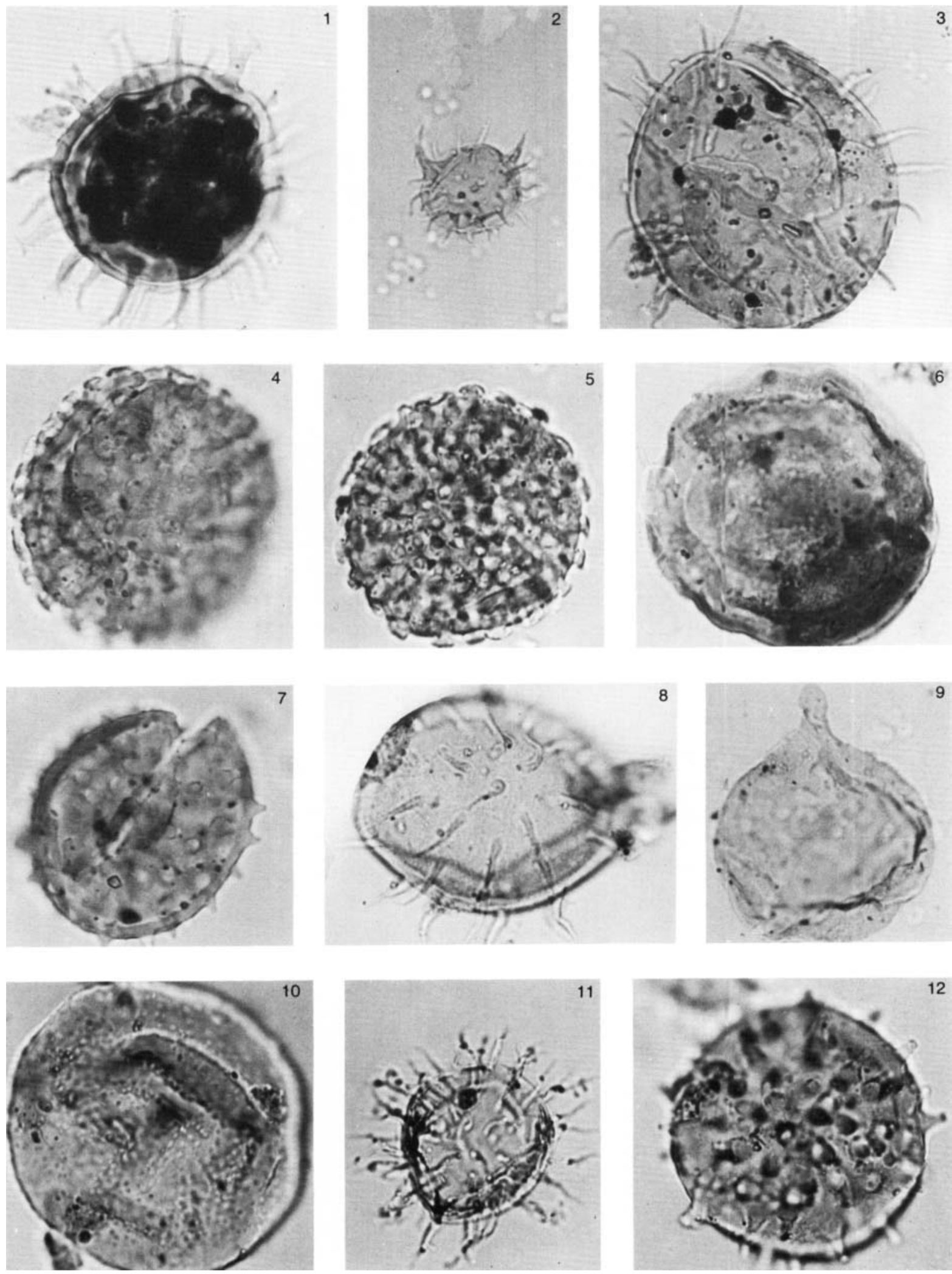